ACCEPTED MANUSCRIPT

\title{
Recurrence coefficients for discrete orthogonal polynomials with hypergeometric weight and discrete Painlevé equations
}

To cite this article before publication: Anton Dzhamay et al 2020 J. Phys. A: Math. Theor. in press https://doi.org/10.1088/1751-8121/abbd54

\section{Manuscript version: Accepted Manuscript}

Accepted Manuscript is "the version of the article accepted for publication including all changes made as a result of the peer review process, and which may also include the addition to the article by IOP Publishing of a header, an article ID, a cover sheet and/or an 'Accepted Manuscript' watermark, but excluding any other editing, typesetting or other changes made by IOP Publishing and/or its licensors"

This Accepted Manuscript is @ 2020 IOP Publishing Ltd.

During the embargo period (the 12 month period from the publication of the Version of Record of this article), the Accepted Manuscript is fully protected by copyright and cannot be reused or reposted elsewhere.

As the Version of Record of this article is going to be / has been published on a subscription basis, this Accepted Manuscript is available for reuse under a CC BY-NC-ND 3.0 licence after the 12 month embargo period.

After the embargo period, everyone is permitted to use copy and redistribute this article for non-commercial purposes only, provided that they adhere to all the terms of the licence https://creativecommons.org/licences/by-nc-nd/3.0

Although reasonable endeavours have been taken to obtain all necessary permissions from third parties to include their copyrighted content within this article, their full citation and copyright line may not be present in this Accepted Manuscript version. Before using any content from this article, please refer to the Version of Record on IOPscience once published for full citation and copyright details, as permissions will likely be required. All third party content is fully copyright protected, unless specifically stated otherwise in the figure caption in the Version of Record.

View the article online for updates and enhancements. 


\title{
Recurrence coefficients for discrete orthogonal polynomials with hypergeometric weight and discrete Painlevé equations
}

\section{Anton Dzhamay}

School of Mathematical Sciences, The University of Northern Colorado, Greeley, CO 80526, USA

E-mail: adzham@unco.edu

\section{Galina Filipuk}

Faculty of Mathematics, Informatics and Mechanics, University of Warsaw, Banacha 2, Warsaw, 02-097, Poland

E-mail: filipuk@mimuw.edu.pl

Alexander Stokes

Department of Mathematics, University College London, Gower Street, London, WC1E 6BT, UK E-mail: alexander.stokes.140ucl.ac.uk

Keywords: orthogonal polynomial ensembles, Askey-Wilson scheme, Painlevé equations, difference equations, isomonodromic transformations, birational transformations.

MSC2010: 33D45, 34M55, 34M56, 14E07, 39A13

\begin{abstract}
Over the last decade it has become clear that discrete Painlevé equations appear in a wide range of important mathematical and physical problems. Thus, the question of recognizing a given non-autonomous recurrence as a discrete Painlevé equation and/determining its type according to Sakai's classification scheme, understanding whether it is equivalent to some known (model) example, and especially finding an explicit change of coordinates transforming it to such an example, becomes one of the central ones. Fortunately, Sakai's geometric theory provides an almost algorithmic procedure for answering this question. In this paper we illustrate this procedure by studying an example coming from the theory of discrete orthogonal polynomials. There are many connections between orthogonal polynomials and Painlevé equations, both differential and discrete. In particular, often the coefficients of three-term recurrence relations for discrete orthogonal polynomials can be expressed in terms of solutions of discrete Painlevé equations. In this work we study discrete orthogonal polynomials with general hypergeometric weight and show that their recurrence coefficients satisfy, after some change of variables, the standard discrete Painlevé-V equation. We also provide an explicit change of variables transforming this equation to the standard form.
\end{abstract}

\section{Introduction}

In describing interesting physical and mathematical models we often rely on various special functions, such as Airy or Bessel functions. Such functions satisfy certain linear ordinary differential equations, and over a hundred years ago P. Painlevé became interested in the question of whether it may be possible to define purely nonlinear special functions as solutions of nonlinear ordinary differential equations. As usual, the nonlinear case is quite subtle, since solutions of nonlinear differential equations do not satisfy the superposition principle, and in general, it may not even be possible to define the notion of a general solution since solutions can develop unexpected singularities that depend not just on the equation, but also on the initial conditions. Nevertheless, this line of reasoning led Painlevé to define a property of an algebraic nonlinear ordinary differential equation (essentially the absence of movable, i.e., dependent on initial conditions, critical singular points) that guarantees the existence of a general solution; this property is now called the Painlevé property. Painlevé and his student B. Gambier then studied a large class of algebraic second-order differential equations that satisfy this property and found that, in addition to equations that are linear or can be reduced to linear, there are six new families of equations that are now called Painlevé equations $\mathrm{P}_{\mathrm{I}}, \ldots, \mathrm{P}_{\mathrm{VI}}$. Solutions of these equations, the so-called Painlevé transcendents, are indeed new purely nonlinear special functions. Over the last fifty years Painlevé transcendents have been playing an increasingly important role

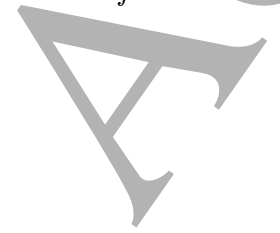


in the description of many nonlinear models, from Quantum Cohomology to the theory of Random Matrices. Probably the most important example is the famous Tracy-Widom distribution from Random Matrix Theory that can be expressed in terms of the Hastings-McLeod solution of the Painlevé-II equation.

The theory of discrete Painlevé equations is much more recent. These equations were originally defined as second-order discrete non-linear equations (or second-order nonlinear recurrence relations) that become one of the differential Painlevé equations in a continuous limit. The intensive study of these equations began in the early 1990's [RGH91] and many examples were obtained in the works of B. Grammaticos, A. Ramani, and their collaborators by the application of the singularity confinement criterion to deautonomizations of known discrete dynamical systems, see the review [GR04] and references therein. Discrete Painlevé equations were also studied from the perspective of the representation theory of affine Weyl groups in a series of papers by M. Noumi, Y. Yamada, and their collaborators, see, e.g, [NY98]. In 2001 H. Sakai, in his seminal paper [Sak01] that used techniques from birational algebraic geometry, gave the definitive classification scheme for discrete Painlevé equations and clarified the relationship between discrete and differential Painlevé equations. Since then the theory of discrete Painlevé equations has reached a certain level of maturity. We know many examples of discrete Painlevé equations, their properties, special solutions for certain parameter values, Lax pairs, various degenerations, etc.; the recent survey paper [KNY17] is both an excellent introduction and a comprehensive overview of the present theory of discrete Painlevé equations.

Moreover, there is an increasing body of evidence that discrete Painlevé equations, similar to their differential counterpart, appear in a wide variety of important applied problems, such as the computations of gap probabilities [Bor03] of various ensembles in the emerging field of integrable probability [BG16], or in describing recurrence coefficients of semi-classical orthogonal polynomials [VA18], and many others. To make a connection between an applied problem and the wealth of known results, it is then important to be able to answer the following sequence of questions:

(a) Suppose one obtains a certain non-linear second order recurrence relation. Does this recurrence fit into the discrete Painlevé framework, i.e., into Sakai's classification scheme?

(b) If so, what is the type of this equation, i.e., the type of its algebraic surface in Sakai's classification?

(c) After the type of the equation is determined, the next question is whether it is equivalent to any known examples of equations of the same type. In general, there are infinitely many non-equivalent discrete Painlevé equations, but usually some simplest forms of such equations are well-known. For example, canonical examples of equations of each type are listed in [KNY17], see also Sakai's original paper [Sak01].

(d) Finally, if the equation is indeed equivalent to a canonical form of some discrete Painlevé equation, how to find an explicit change of variables transforming one equation into the other. In particular, answering this question requires matching of various parameters in the applied problem with parameters in the standard form of this discrete Painlevé equation. Note also that being able to do this may also result in uncovering new connections between very different problems.

Fortunately, the algebro-geometric theory of Painlevé equations provides us with a powerful set of tools and essentially a near-algorithmic procedure to answer exactly these questions. Unfortunately, the necessary mathematical background to master this theory, such as birational algebraic geometry, the representation theory of affine Weyl groups, and the word equivalence problem in groups, is often quite different from that of the researchers working with applied problems, and so the learning curve can feel steep. Nevertheless, we believe that it is still possible to learn, at least on a computational level first, the essentials of how to approach these questions. Thus, the purpose of the present paper is to illustrate the above procedure in detail using one concrete example, hoping that anyone interested would then be able to make necessary changes to adjust this procedure for a different example.

The problem that we consider belongs to the theory of orthogonal polynomials. In fact, the relationship between discrete Painlevé equations and orthogonal polynomials is much older than the actual definition of a discrete Painlevé equation — the first example of a discrete Painlevé-I equation originally appeared in 
the work of Shohat [Sho39]. There are many connections between recurrence coefficients of semi-classical orthogonal polynomials and solutions of Painlevé equations, both discrete and differential (see, for instance, [VA18] and numerous references therein).

Let $\left\{p_{n}(x)=\gamma_{n} x^{n}+\cdots\right\}$ be the collection of polynomials that are orthonormal on the set $\mathbb{N}=\{0,1,2, \ldots\}$ of non-negative integers with respect to the hypergeometric weight $w_{k}$,

$$
\sum_{k=0}^{\infty} p_{n}(k) p_{m}(k) w_{k}=\delta_{m, n}, \quad w_{k}=\frac{(\alpha)_{k}(\beta)_{k}}{(\gamma)_{k} k !} c^{k}, \quad \alpha, \beta, \gamma>0,0<c<1
$$

where $(\cdot)_{k}$ is the usual Pochhammer symbol and $\delta_{m, n}$ is the Kronecker delta. This collection of polynomials is known as the discrete orthogonal polynomials with hypergeometric weights, since the moments of this weight function are given in terms of the Gauss hypergeometric function ${ }_{2} F_{1}(\alpha, \beta ; \gamma ; c)$ and its derivatives; it has been recently studied in [FVA18, Dom16]. These polynomials satisfy the three term recurrence relation

$$
x p_{n}(x)=a_{n+1} p_{n+1}(x)+b_{n} p_{n}(x)+a_{n} p_{n-1}(x)
$$

where $a_{0}=0$. The coefficients $a_{n}$ and $b_{n}$ are called the recurrence coefficients [Chi78, Ism05, Sze67]. Note that the corresponding monic orthogonal polynomials $P_{n}=p_{n} / \gamma_{n}$ satisfy a similar three term recurrence relation

$$
x P_{n}(x)=P_{n+1}(x)+b_{n} P_{n}(x)+a_{n}^{2} P_{n-1}(x) .
$$

In [FVA18] it was shown that these recurrence coefficients $\left\{a_{n}, b_{n}\right\}$, as functions of the discrete variable $n$, satisfy, after some change of variables, a system of non-linear difference equations and as functions of the continuous parameter $c$ of the hypergeometric weight, they satisfy the differential Toda system. From the differential and discrete systems one can obtain a differential equation, which in turn can be reduced to the $\sigma$-form of the sixth Painlevé equation. In [HFC19], using a direct computation, it was then shown that the discrete system is a composition of Bäcklund transformations of the sixth Painlevé equation. In the present paper we give a geometric explanation of this result, show that the discrete system is in fact equivalent to the standard discrete Painlevé-V equation, and provide an explicit change of variables achieving that.

To be more specific, let us introduce two new variables $x_{n}$ and $y_{n}$ parameterizing the recurrence coefficients $a_{n}^{2}$ and $b_{n}$ via

$$
a_{n}^{2} \frac{1-c}{c}=y_{n}+\sum_{k=0}^{n-1} x_{k}+\frac{n(n+\alpha+\beta-\gamma-1)}{1-c}, \quad b_{n}=x_{n}+\frac{n+(n+\alpha+\beta) c-\gamma}{1-c}
$$

It was shown in [FVA18, Theorem 3.1] that $x_{n}, y_{n}, n \in \mathbb{N}$, satisfy the first-order system of non-linear non-autonomous difference equations

$$
\begin{aligned}
& \left(y_{n}-\alpha \beta+(\alpha+\beta+n) x_{n}-x_{n}^{2}\right)\left(y_{n+1}-\alpha \beta+(\alpha+\beta+n+1) x_{n}-x_{n}^{2}\right) \\
& \quad=\frac{1}{c}\left(x_{n}-1\right)\left(x_{n}-\alpha\right)\left(x_{n}-\beta\right)\left(x_{n}-\gamma\right), \\
& \left(x_{n}+\mathfrak{Y}_{n}\right)\left(x_{n-1}+\mathfrak{Y}_{n}\right) \\
& \quad=\frac{\left(y_{n}+n \alpha\right)\left(y_{n}+n \beta\right)\left(y_{n}+n \gamma-(\gamma-\alpha)(\gamma-\beta)\right)\left(y_{n}+n-(1-\alpha)(1-\beta)\right)}{\left(y_{n}(2 n+\alpha+\beta-\gamma-1)+n((n+\alpha+\beta)(n+\alpha+\beta-\gamma-1)-\alpha \beta+\gamma)\right)^{2}},
\end{aligned}
$$

where $\alpha, \beta, \gamma, c$ are the parameters of the hypergeometric weight $w_{k}$ in (1.1) and

$$
\mathfrak{Y}_{n}=\frac{y_{n}^{2}+y_{n}(n(n+\alpha+\beta-\gamma-1)-\alpha \beta+\gamma)-\alpha \beta n(n+\alpha+\beta-\gamma-1)}{y_{n}(2 n+\alpha+\beta-\gamma-1)+n((n+\alpha+\beta)(n+\alpha+\beta-\gamma-1)-\alpha \beta+\gamma)} .
$$

The initial conditions for this recurrence are given by

$$
x_{0}=\frac{\alpha \beta c}{\gamma} \frac{{ }_{2} F_{1}(\alpha+1, \beta+1 ; \gamma+1 ; c)}{{ }_{2} F_{1}(\alpha, \beta ; \gamma ; c)}+\frac{(\alpha+\beta) c-\gamma}{c-1}, \quad y_{0}=0 .
$$


For the hypergeometric weights the connection with the $\sigma$-form of the sixth Painlevé equation (with independent variable $c$ ) is known (see [FVA18, Theorem 5.1]). The essential role is played by the Toda system for the recurrence coefficients (see, e.g., [Ism05, §2.8] or [VA18, §3.2.2]). For the hypergeometric weights, it is given by

$$
\begin{aligned}
c \frac{d}{d c} a_{n}^{2} & =a_{n}^{2}\left(b_{n}-b_{n-1}\right), \quad n \geq 1, \\
c \frac{d}{d c} b_{n} & =a_{n+1}^{2}-a_{n}^{2}, \quad n \geq 0 .
\end{aligned}
$$

It is proved in [FVA18, Theorem 5.1] that a simple linear change of variable transforms $S_{n}=\sum_{k=0}^{n-1} x_{k}$ into the solutions of the $\sigma$-form of the sixth Painlevé equation. Knowing $S_{n}$ one can find $x_{n}$, $y_{n}$ and, hence, the recurrence coefficients $a_{n}^{2}, b_{n}$ in terms of $S_{n}$ and its derivatives. Moreover, it is shown in [HFC19] that the differential equation for $x_{n}$ can be directly reduced to the sixth Painlevé equation.

Our main objective for this paper is to illustrate the general process of identifying a discrete dynamical system as a discrete Painlevé equation and explicitly rewriting it in some standard form, using equations (1.5-1.6) as an example of an applied system. This process consists of the following steps, where we assume that we indeed have some discrete Painlevé equation, otherwise the process will terminate at some step.

(Step 1) Identify the singularity structure of the problem. For that, if necessary, rewrite our recurrence equation as a system of two first-order recurrences, $\left(x_{n+1}, y_{n+1}\right)=\psi^{(n)}\left(x_{n}, y_{n}\right)$. The mapping $\psi^{(n)}: \mathbb{C}^{2} \rightarrow \mathbb{C}^{2}$ should be a birational mapping that may depend on various parameters, including the iteration step $n$ that we consider to be generic. Then compactify the configuration space from $\mathbb{C}^{2}=\mathbb{C} \times \mathbb{C}$ to $\mathbb{P}^{1} \times \mathbb{P}^{1}$. Find the base points of the mapping and resolve them using the blowup procedure. Continue doing that until all base points for both $\psi^{(n)}$ and $\left(\psi^{(n)}\right)^{-1}$ are resolved (for discrete Painlevé equations this process should terminate in finitely many steps). Thus, we get an isomorphism of resulting rational algebraic surfaces, $\psi^{(n)}: X_{n} \stackrel{\simeq}{\rightarrow} X_{n+1}$. In making this computation, it is important to keep in mind that positions of base points usually evolve with the mapping, so one needs to be careful distinguishing between the points in the domain and the points in the range. We also remark that sometimes the singularity structure can be seen before even studying the dynamics; e.g., singularities can occur as a result of a parameterization of some moduli space appearing in the problem, as in [DK19], for example.

(Step 2) Linearize the mapping on $\operatorname{Pic}(\mathcal{X})$. This can be done explicitly in relatively simple cases, such as the present example. But sometimes the evolution mapping can be too complicated even for a Computer Algebra System. In this case, it may be possible to deduce the action of the mapping on $\operatorname{Pic}(X)$ from the knowledge of parameter evolution via the Period Map, see [DK19] for an example of such a computation.

(Step 3) Determine the surface type, according to Sakai's classification scheme. For a discrete Painlevé equation, although the positions of base points may evolve, the configuration will stay fixed, and so the surfaces $\left\{X_{n}\right\}$ will all have the same type. There should be eight such base points; those points will lie on some (generically unique) biquadratic curve on $\mathbb{P}^{1} \times \mathbb{P}^{1}$ (i.e., a curve whose defining polynomial, when written in a coordinate chart, has bi-degree $(2,2))$ and the point configuration is defined/to be the configuration of the irreducible components of this curve. Each such component should have self-intersection index -2 and is associated with a node of an affine Dynkin diagram; nodes are connected when the corresponding components intersect. The type of this Dynkin diagram is called the surface type of the equation. This description assumes that the surfaces $x_{n}$ are minimal, but it can happen that after the initial blowup procedure is complete, some -1-curves would have to be blown down. This will also result in some irreducible components having higher negative self-intersection index. The blowing down procedure is quite delicate, so here we assume that the surfaces $\mathcal{X}_{n}$ are indeed minimal, but see [DST13] and [DK19] for examples requiring a blowing down.

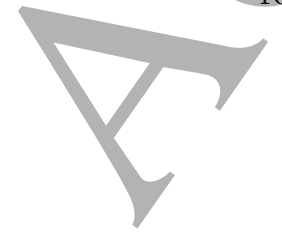


(Step 4) Find a preliminary change of basis of $\operatorname{Pic}(\mathcal{X})$. At this step, we only need to ensure that this change of basis identifies the surface roots (or nodes of the Dynkin diagrams of our surface) with the standard example.

(Step 5) Find the translation vector and compare it with the standard dynamic. Using this preliminary change of basis we can define the symmetry roots for our surface that match the standard example. Using the action $\varphi_{*}$ of the mapping on $\operatorname{Pic}(X)$ we can then see the induced action on the symmetry sub-lattice and, in particular, on the symmetry roots. For the discrete Painlevé equations, this action on each root should be a translation by some multiple of the anticanonical divisor class. Even when this vector is not the same as the translation/vector for the reference dynamic, it may be conjugate to it. To find out whether this is the case, we represent each translation as a word in the generators of the extended affine Weyl group and solve the conjugacy problem for words in groups. If they are conjugate, the conjugation element is the necessary adjustment to our preliminary change of basis.

(Step 6) Find the change of variables reducing the applied problem to the standard example. Adjusting the change of bases in $\operatorname{Pic}(\mathcal{X})$, if necessary, we now have the identification on the level of the Picard lattice. Next, we need to find the actual change of variables that induces that linear change of basis. For that, identify the curves that form the basis in the corresponding coordinate pencils. Those curves then are our projective coordinates, up to a Möbius transformation. To fix the Möbius transformations, use the mapping of coordinate divisors. An important part of this computation is the identification of various parameters between the two problems. This, in fact, can be done ahead of time by using the Period Map, which gives the parameterization in terms of canonical (for the given choice of root bases) root variables. Expressing these root variables in terms of parameters of the problem gives the necessary identification of parameters.

In the next section we carefully illustrate each step of this procedure using equations (1.5-1.6) as an example of an applied system. Our main result is the following Theorem.

Theorem 1. Recurrences (1.5-1.6) are equivalent to the standard discrete Painlevé equation (A.19). This equivalence is achieved via the following change of variables:

$$
\begin{aligned}
x(f, g)=\gamma & -g-\frac{(n+\beta) f}{f-1}, \\
y(f, g)=(g+\alpha+\beta+n-\gamma)(g+2 \beta+2 n-\gamma)-n \alpha-\frac{g t(g+\beta-\gamma)}{f} & \\
& +\frac{(n+\beta)((c-1)(2 g+\alpha+3 \beta+3 n-2 \gamma)+(\alpha+\beta+n-\gamma)+n)}{c(f-1)}+\frac{(c-1)(n+\beta)^{2}}{c(f-1)^{2}} .
\end{aligned}
$$

The inverse change of variables is given by

$$
\begin{aligned}
f(x, y) & =\frac{t(x-\beta)(x-\gamma)}{((x-\alpha)(x-\beta)-n x-y)}, \\
g(x, y) & =-\frac{(x-\gamma)(((x-\alpha)(x-\beta)-n x-y)-t(x-\beta)(x-\gamma+\beta+n))}{((x-\alpha)(x-\beta)-n x-y)-t(x-\beta)(x-\gamma)} .
\end{aligned}
$$

Note that the parameters $c$ and $t$ are related by $c t=1$, and the relationship between parameters $\alpha, \beta, \gamma, n$ and the root variables $a_{0}, \ldots a_{4}$ in the standard example is given by (2.14).

The standard difference Painlevé-V equation is one of the equations in the d-P $\left(\mathrm{D}_{4}^{(1)} / \mathrm{D}_{4}^{(1)}\right)$ family of discrete Painlevé equations whose geometric (i.e., point configuration) and algebraic (extended affine Weyl symmetry group) data are both encoded by affine Dynkin diagrams of type $D_{4}^{(1)}$; we collect some basic facts and data about this family in the Appendix (see also [KNY17]). 


\section{The Identification Procedure}

\subsection{The Singularity Structure}

The first step in the geometric analysis of discrete Painlevé equations is to understand the singularity structure of the system (which is best done with the help of some Computer Algebra System; in this project we used Mathematica $\left.{ }^{\circledR}\right)$.

Note that equation (1.5) defines the forward mapping $\psi_{1}^{(n)}:\left(x_{n}, y_{n}\right) \mapsto\left(x_{n}, y_{n+1}\right)$ and equation (1.6) defines the backward mapping $\psi_{2}^{(n)}:\left(x_{n}, y_{n}\right) \mapsto\left(x_{n-1}, y_{n}\right)$, this is fairly typical for discrete Painlevé equations obtained as deautonomizations of QRT mappings, see [CDT17].

First, we compactify the affine complex plane $\mathbb{C}^{2}$ to $\mathbb{P}^{1} \times \mathbb{P}^{1}$ by introducing homogeneous coordinates $\left[x^{0}: x^{1}\right]$ and $\left[y^{0}: y^{1}\right]$ with $x=x^{0} / x^{1}$ in the affine chart $x_{1} \neq 0, X=1 / x=x^{1} / x^{0}$ in the affine chart $x_{0} \neq 0$ and $y$ and $Y=1 / y$ defined similarly. Next, we look for indeterminacies of rational maps, i.e., the points $q_{i}$ where both the numerator and the denominator of the map vanish. At those points we perform the blowup procedure to resolve such indeterminacies.

Let us now very briefly recall the blowup procedure, see, e.g., [Sha13] for details, that is particularly simple in the two-dimensional case that we consider. Geometrically, the blowup procedure "separates" the lines passing through the point $q_{i}$ (the center of the blowup) by "lifting" them according to their "slopes" (see the left picture on Figure 1 for the local illustration of a blowup in the real-variable case). Topologically, for complex surfaces, blowup is a surgery that creates a Riemann sphere "bubble" (projectivized tangent space) $S^{2} \simeq \mathbb{P}_{\mathbb{C}}^{1}$ in place of the center of the blowup $q_{i}$, thus adding a new spherical class to homology (and, via the Poincaré duality, cohomology) of the surface. Algebraically, the blowup procedure is an introduction of two new charts $\left(u_{i}, v_{i}\right)$ and $\left(U_{i}, V_{i}\right)$ in the neighborhood of the blowup point $q_{i}\left(x_{i}, y_{i}\right)$, where the change of variables is given by $x=x_{i}+u_{i}=x_{i}+U_{i} V_{i}$ and $y=y_{i}+u_{i} v_{i}=y_{i}+V_{i}$. This change of variables is a bijection away from $q_{i}$, but the point $q_{i}$ is replaced by the $\mathbb{P}^{1}$-line of all possible slopes, called the central fiber or the exceptional divisor of the blowup. We denote this central fiber by $F_{i}$ (and sometimes by $E_{i}$ ), it is given in the blowup charts by local equations $u_{i}=0$ and $V_{i}=0$. For these charts the upper/lower-case naming convention is only for convenience and, in contrast to the naming of affine charts, it does not hold that $U_{i}=1 / u_{i}$. However, it is true that $v_{i}=1 / U_{i}$ - these local coordinates on $\mathbb{P}^{1}$ represent all possible slopes of lines passing through the point $q_{i}$, and so this variable change "separates" all curves passing through $q_{i}$ based on their slopes. Schematically, it is convenient to illustrate the blowup on a diagram as shown on the right on Figure 1. The notation $L-F_{i}$ denotes the proper transform $\overline{\pi^{-1}\left(L-\left(x_{i}, y_{i}\right)\right)}$, that needs to be distinguished from the total transform $\pi^{-1}(L)=\left(L-F_{i}\right)+F_{i}$. Note that, despite the presence of the negative sign, $L-F_{i}$ is an actual geometric curve, i.e., an effective divisor.

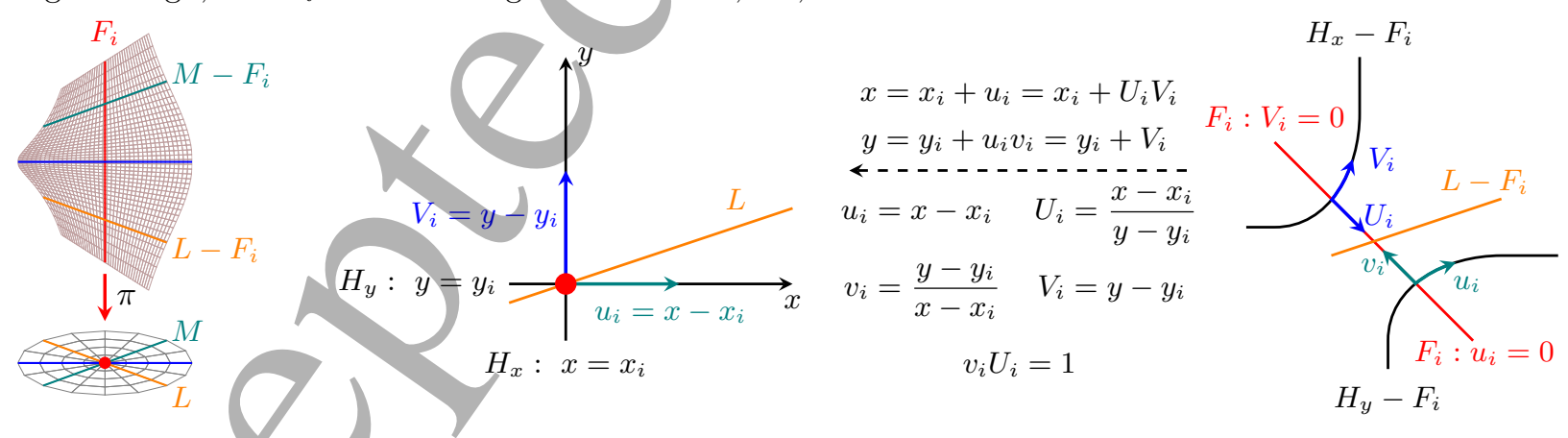

Figure 1: The Blowup Procedure 


\subsubsection{The Forward Mapping}

We begin by considering the forward mapping. We put $\bar{x}=x:=x_{n}, y:=y_{n}, \bar{y}:=y_{n+1}$ and omit the index $n$ in the mapping notation. The map $\psi_{1}:(x, y) \mapsto(\bar{x}, \bar{y})$ then becomes

$$
(\bar{x}, \bar{y})=\left(x, \frac{(x-1)(x-\alpha)(x-\beta)(x-\gamma)}{c(y-(x-\alpha)(x-\beta)+n x)}+(x-\alpha)(x-\beta)-(n+1) x\right)
$$

and we immediately see the following base points (in the affine coordinates $(x, y)$ ):

$$
q_{1}(1,(1-\alpha)(1-\beta)-n), \quad q_{2}(\alpha,-n \alpha), \quad q_{3}(\beta,-n \beta), \quad q_{4}(\gamma,(\gamma-\alpha)(\gamma-\beta)-n \gamma) .
$$

Rewriting the mapping for $\bar{y}$ in the $(X, Y)$-chart, we get

$$
\bar{y}=\frac{\left(\begin{array}{r}
Y(1-X)(1-\alpha X)(1-\beta X)(1-\gamma X) \\
+c\left(X^{2}-Y((1-\alpha X)(1-\beta X)-n X)\right)((1-\alpha X)(1-\beta X)-(n+1) X)
\end{array}\right)}{c X^{2}\left(X^{2}-Y((1-\alpha X)(1-\beta X)-n X)\right)},
$$

we see that we get a new base point $q_{5}(x=\infty, y=\infty)$ or $q_{5}(X=0, Y=0)$. It is easy to see that these points are the only base points on $\mathbb{P}^{1} \times \mathbb{P}^{1}$ for the forward dynamic. Thus, if this mapping is indeed in the discrete Painlevé family, there are three more points on exceptional divisors (these points can also appear for the backward dynamic, but we show later that this is not the case).

Resolving $q_{1}, \ldots, q_{4}$. We introduce blowup coordinates at $q_{1}(1,(1-\alpha)(1-\beta)-n)$ via

$$
x=1+u_{1}=1+U_{1} V_{1}, \quad y=(1-\alpha)(1-\beta)-n+u_{1} v_{1}=(1-\alpha)(1-\beta)-n+V_{1} .
$$

In the coordinates $\left(u_{1}, v_{1}\right)$ we get

$$
\bar{x}=1+u_{1}, \quad \bar{y}=\frac{u_{1}\left(1-\alpha+u_{1}\right)\left(1-\beta+u_{1}\right)\left(1-\gamma+u_{1}\right)}{c u_{1}\left(v_{1}-\left(2-\alpha-\beta+u_{1}\right)+n\right)}+\left(1-\alpha+u_{1}\right)\left(1-\beta+u_{1}\right)-(n+1)\left(1+u_{1}\right),
$$

and we first see that the cancelation of $u_{1}$ in the fraction resolves the indeterminacy, and so the mapping lifts to the exceptional divisor $F_{1}$ whose equation in this chart is $u_{1}=0$. Studying the mapping in the $\left(U_{1}, V_{1}\right)$-chart does not give any new information.

The computation is exactly the same for the points $q_{2}, \ldots, q_{4}$, the mapping extends without new base points to the exceptional divisors $F_{i}$.

Resolving $q_{5}$ and its degeneration cascade. The situation at the point $q_{5}(\infty, \infty)$ is more interesting. Introducing blowup coordinates at this point via

$$
X=u_{5}=U_{5} V_{5}, \quad Y=u_{5} v_{5}=V_{5},
$$

and considering the mapping in the $\left(u_{5}, v_{5}\right)$-chart, we get, after cancelling the $u_{5}$-factor in the numerator and denominator,

$$
\bar{y}\left(u_{5}, v_{5}\right)=\frac{\left(\begin{array}{r}
v_{5}\left(1-u_{5}\right)\left(1-\alpha u_{5}\right)\left(1-\beta u_{5}\right)\left(1-\gamma u_{5}\right) \\
+c\left(u_{5}-v_{5}\left(\left(1-\alpha u_{5}\right)\left(1-\beta u_{5}\right)-n u_{5}\right)\right)\left(\left(1-\alpha u_{5}\right)\left(1-\beta u_{5}\right)-(n+1) u_{5}\right)
\end{array}\right)}{c u_{5}^{2}\left(u_{5}-v_{5}\left(\left(1-\alpha u_{5}\right)\left(1-\beta u_{5}\right)-n u_{5}\right)\right)} .
$$


We see that this mapping has a new base point $q_{6}\left(u_{5}=X=0, v_{5}=Y / X=0\right)$ (note that this base point is not visible in the $\left(U_{5}, V_{5}\right)$-chart). Continuing in this way, we get the following cascade of "infinitely close" base points:

$$
\begin{aligned}
q_{5}(X=0, Y=0) & \leftarrow q_{6}\left(u_{5}=X=0, v_{5}=\frac{Y}{X}=0\right) \leftarrow q_{7}\left(u_{6}=u_{5}=0, v_{6}=\frac{v_{5}}{u_{5}}=\frac{c}{c-1}\right) \\
& \leftarrow q_{8}\left(u_{7}=u_{6}=0, v_{7}=\frac{(c-1) v_{6}-c}{(c-1) u_{6}}=\frac{c(c(\alpha+\beta+n)+n-\gamma)}{(c-1)^{2}}\right) .
\end{aligned}
$$

Note that the positions of base points depend on $n$, and so evolve with the dynamics, but the configuration of base points remains fixed. Put $X_{n}:=\mathrm{Bl}_{q_{1} \cdots q_{8}}\left(\mathbb{P}^{1} \times \mathbb{P}^{1}\right)$ and let $\eta_{n}: X_{n} \rightarrow \mathbb{P}^{1} \times \mathbb{P}^{1}$ be the corresponding blow down map. This gives us a typical surface in the family on which the dynamic is defined. In what follows we may sometimes omit the index $n$ when only the point configuration and not the exact location of the base points is important.

\subsubsection{The Backward Mapping}

Consider now the backward mapping. We put $x:=x_{n}, \underline{x}=x_{n-1}, \underline{y}=y:=y_{n}$, The backward mapping $\psi_{2}:(x, y) \mapsto(\underline{x}, \underline{y})$ then becomes

$$
(\underline{x}, \underline{y})=\left(\frac{(y+n \alpha)(y+n \beta)(y+n \gamma-(\gamma-\alpha)(\gamma-\beta))(y-n-(1-\alpha)(1-\beta))}{(x+\mathfrak{Y})(y(2 n+\alpha+\beta-\gamma-1)+n((n+\alpha+\beta)(n+\alpha+\beta-\gamma-1)-\alpha \beta+\gamma))^{2}}-\mathfrak{Y}, y\right),
$$

where $\mathfrak{Y}$ is given by (1.7) (we omit the index $n$ ). The same standard computation shows that the only base points of the backwards dynamic are the same points $q_{1}, \ldots, q_{4}$ as for the forward dynamic, but the singularity cascade at $q_{5}$ is not present.

\subsection{The Mapping on $\operatorname{Pic}(X)$}

Recall that for a regular algebraic variety $X$, its Picard group (or Picard lattice) is the quotient of the divisor group $\operatorname{Div}(X)=\operatorname{Span}_{\mathbb{Z}}(\mathcal{D})$, that is a free Abelian group generated by closed irreducible subvarieties $D$ of codimension 1, by the subgroup $\mathrm{P}(X)$ of principal divisors (i.e., by the relation of linear equivalence),

$$
\operatorname{Pic}(x) \simeq \operatorname{Cl}(x)=\operatorname{Div}(x) / \mathrm{P}(x)=\operatorname{Div}(x) / \sim,
$$

see [SKKT00] or [Sha13]. In our case, it is enough to know that $\operatorname{Pic}\left(\mathbb{P}^{1} \times \mathbb{P}^{1}\right)=\operatorname{Span}_{\mathbb{Z}}\left\{\mathcal{H}_{x}, \mathcal{H}_{y}\right\}$, where $\mathcal{H}_{x}=\left[H_{x=a}\right]$ is the class of a vertical and $\mathcal{H}_{y}=\left[H_{y=b}\right]$ is the class of a horizontal line on $\mathbb{P}^{1} \times \mathbb{P}^{1}$. Each blowup procedure at a point $q_{i}$ adds the class $\mathcal{F}_{i}=\left[F_{i}\right]$ of the exceptional divisor (i.e., the central fiber) of the blowup, so $\operatorname{Pic}\left(X_{n}\right)=\operatorname{Span}_{\mathbb{Z}}\left\{\mathcal{H}_{x}, \mathcal{H}_{y}, \mathcal{F}_{1}, \ldots, \mathcal{F}_{8}\right\}$. Further, the Picard lattice is equipped with the symmetric bilinear intersection form given by

$$
\mathcal{H}_{x} \bullet \mathcal{H}_{x}^{\widehat{C}}=\mathcal{H}_{y} \bullet \mathcal{H}_{y}=\mathcal{H}_{x} \bullet \mathcal{F}_{i}=\mathcal{H}_{y} \bullet \mathcal{F}_{j}=0, \quad \mathcal{H}_{x} \bullet \mathcal{H}_{y}=1, \quad \mathcal{F}_{i} \bullet \mathcal{F}_{j}=-\delta_{i j}
$$

on the generators, and then extended by linearity.

Both the forward and the backward mappings induce linear maps on $\operatorname{Pic}(X)$. We use the notation $\operatorname{Pic}(\bar{X})$ (resp. Pic $(\underline{X}))$ for the range of the forward (resp. backward) mappings; note that all these groups are clearly canonically isomorphic, so we sometimes just use the notation $\operatorname{Pic}(X)$. We use $\bar{F}_{i}$ to denote the divisor of the central fiber of the blowup at the point $\bar{q}_{i}=\psi_{1}\left(q_{i}\right)$, and similarly for the backwards mapping and for the classes.

Since the mapping is not very complicated, we can compute its action on $\operatorname{Pic}(X)$ directly. The result is given by the following Lemma, where we use the notation $\mathcal{F}_{i j}=\mathcal{F}_{i}+\mathcal{F}_{j}$ and so on. 


\section{Lemma 2.}

(a) The action of the forward dynamic $\left(\psi_{1}\right)_{*}: \operatorname{Pic}(\mathcal{X}) \rightarrow \operatorname{Pic}(\bar{X})$ is given by

$$
\begin{aligned}
& \mathcal{H}_{x} \mapsto \overline{\mathcal{H}}_{x}, \quad \mathcal{F}_{1} \mapsto \overline{\mathcal{H}}_{x}-\overline{\mathcal{F}}_{1}, \quad \mathcal{F}_{3} \mapsto \overline{\mathcal{H}}_{x}-\overline{\mathcal{F}}_{3}, \quad \mathcal{F}_{5} \mapsto \overline{\mathcal{H}}_{x}-\overline{\mathcal{F}}_{8}, \quad \mathcal{F}_{7} \mapsto \overline{\mathcal{H}}_{x}-\overline{\mathcal{F}}_{6}, \\
& \mathcal{H}_{y} \mapsto 4 \overline{\mathcal{H}}_{x}+\overline{\mathcal{H}}_{y}-\overline{\mathcal{F}}_{12345678}, \quad \mathcal{F}_{2} \mapsto \overline{\mathcal{H}}_{x}-\overline{\mathcal{F}}_{2}, \quad \mathcal{F}_{4} \mapsto \overline{\mathcal{H}}_{x}-\overline{\mathcal{F}}_{4}, \quad \mathcal{F}_{6} \mapsto \overline{\mathcal{H}}_{x}-\overline{\mathcal{F}}_{7}, \quad \mathcal{F}_{8} \mapsto \overline{\mathcal{H}}_{x}-\overline{\mathcal{F}}_{5},
\end{aligned}
$$

and the evolution of base points $\bar{q}_{i}=\psi_{1}\left(q_{i}\right)$ is given by

$$
\bar{q}_{1}(1,(1-\alpha)(1-\beta)-(n+1)), \bar{q}_{2}(\alpha,-(n+1) \alpha), \bar{q}_{3}(\beta,-(n+1) \beta), \bar{q}_{4}(\gamma,(\gamma-\alpha)(\gamma-\beta)-(n+1) \gamma)
$$

for finite points, and for the degeneration cascade we get

$$
\begin{aligned}
\bar{q}_{5}(\bar{X}=0, \bar{Y}=0) & \leftarrow \bar{q}_{6}\left(\bar{u}_{5}=\bar{X}=0, \bar{v}_{5}=\frac{\bar{Y}}{\bar{X}}=0\right) \leftarrow \bar{q}_{7}\left(\bar{u}_{6}=\bar{u}_{5}=0, \bar{v}_{6}=\frac{\bar{v}_{5}}{\bar{u}_{5}}=\frac{c}{c-1}\right) \\
& \leftarrow \bar{q}_{8}\left(\bar{u}_{7}=\bar{u}_{6}=0, \bar{v}_{7}=\frac{(c-1) \bar{v}_{6}-c}{(c-1) \bar{u}_{6}}=\frac{c(c(\alpha+\beta+n+1)+n-\gamma-1)}{(c-1)^{2}}\right) .
\end{aligned}
$$

(b) The action of the backwards dynamic $\left(\psi_{2}\right)_{*}: \operatorname{Pic}(X) \rightarrow \operatorname{Pic}(\underline{X})$ is given by

$$
\begin{aligned}
& \mathcal{H}_{x} \mapsto \underline{\mathcal{H}}_{x}+2 \underline{\mathcal{H}}_{y}-\underline{\mathcal{F}}_{1234}, \quad \mathcal{F}_{1} \mapsto \underline{\mathcal{H}}_{y}-\underline{\mathcal{F}}_{1}, \quad \mathcal{F}_{3} \mapsto \underline{\mathcal{H}}_{y}-\underline{\mathcal{F}}_{3}, \quad \mathcal{F}_{5} \mapsto \underline{\mathcal{F}}_{5}, \quad \mathcal{F}_{7} \mapsto \underline{\mathcal{F}}_{7}, \\
& \mathcal{H}_{y} \mapsto \underline{\mathcal{H}}_{y}, \quad \mathcal{F}_{2} \mapsto \underline{\mathcal{H}}_{y}-\underline{\mathcal{F}}_{2}, \quad \mathcal{F}_{4} \mapsto \underline{\mathcal{H}}_{y}-\underline{\mathcal{F}}_{4}, \quad \mathcal{F}_{6} \mapsto \underline{\mathcal{F}}_{6}, \quad \mathcal{F}_{8} \mapsto \underline{\mathcal{F}}_{8} .
\end{aligned}
$$

From this we can also easily compute the evolution of base points. We get

$$
\underline{q}_{1}(1,(1-\alpha)(1-\beta)-n), \underline{q}_{2}(\alpha,-n \alpha), \underline{q}_{3}(\beta,-n \beta), \underline{q}_{4}(\gamma,(\gamma-\alpha)(\gamma-\beta)-n \gamma),
$$

as well as the degeneration cascade

$$
\begin{aligned}
\underline{q}_{5}(\underline{X}=0, \underline{Y}=0) & \leftarrow \underline{q}_{6}\left(\underline{u}_{5}=\underline{X}=0, \underline{v}_{5}=\frac{\underline{Y}}{\underline{X}}=0\right) \leftarrow \underline{q}_{7}\left(\underline{u}_{6}=\underline{u}_{5}=0, \underline{v}_{6}=\frac{\underline{v}_{5}}{\underline{u}_{5}}=\frac{c}{c-1}\right) \\
& \leftarrow \underline{q}_{8}\left(\underline{u}_{7}=\underline{u}_{6}=0, \underline{v}_{7}=\frac{(c-1) \underline{v}_{6}-c}{(c-1) \underline{u}_{6}}=\frac{c(c(\alpha+\beta+n)+n-\gamma-2)}{(c-1)^{2}}\right) .
\end{aligned}
$$

(c) The action of the composed mapping $\psi_{*}^{(n)}=\psi_{*}=\left(\psi_{2}\right)_{*}^{-1} \circ\left(\psi_{1}\right)_{*}$ : $\operatorname{Pic}\left(X_{n}\right) \rightarrow \operatorname{Pic}\left(X_{n+1}\right)$ is given by

$$
\begin{array}{rlrl}
\mathcal{H}_{x} & \mapsto \overline{\mathcal{H}}_{x}+2 \overline{\mathcal{H}}_{y}-\overline{\mathcal{F}}_{1234}, & & \mathcal{H}_{y} \mapsto 4 \overline{\mathcal{H}}_{x}+5 \overline{\mathcal{H}}_{y}-3 \overline{\mathcal{F}}_{1234}-\overline{\mathcal{F}}_{5678}, \\
\mathcal{F}_{1} & \mapsto \overline{\mathcal{H}}_{x}+\overline{\mathcal{H}}_{y}-\overline{\mathcal{F}}_{234}, & & \mathcal{F}_{5} \mapsto \overline{\mathcal{H}}_{x}+2 \overline{\mathcal{H}}_{y}-\overline{\mathcal{F}}_{12348} \\
\mathcal{F}_{2} & \mapsto \overline{\mathcal{H}}_{x}+\overline{\mathcal{H}}_{y}-\overline{\mathcal{F}}_{134}, & & \mathcal{F}_{6} \mapsto \overline{\mathcal{H}}_{x}+2 \overline{\mathcal{H}}_{y}-\overline{\mathcal{F}}_{12347}, \\
\mathcal{F}_{3} & \mapsto \overline{\mathcal{H}}_{x}+\overline{\mathcal{H}}_{y}-\overline{\mathcal{F}}_{124}, & \mathcal{F}_{7} \mapsto \overline{\mathcal{H}}_{x}+2 \overline{\mathcal{H}}_{y}-\overline{\mathcal{F}}_{12346}, \\
\mathcal{F}_{4} \mapsto \overline{\mathcal{H}}_{x}+\overline{\mathcal{H}}_{y}-\overline{\mathcal{F}}_{123}, & \mathcal{F}_{8} \mapsto \overline{\mathcal{H}}_{x}+2 \overline{\mathcal{H}}_{y}-\overline{\mathcal{F}}_{12345} .
\end{array}
$$

The evolution of the base points (here $\bar{q}_{i}=\psi^{(n)}\left(q_{i}\right)$ ) is

$$
\bar{q}_{1}(1,(1-\alpha)(1-\beta)-(n+1)), \bar{q}_{2}(\alpha,-(n+1) \alpha), \bar{q}_{3}(\beta,-(n+1) \beta), \bar{q}_{4}(\gamma,(\gamma-\alpha)(\gamma-\beta)-(n+1) \gamma)
$$

for finite points, and

$$
\begin{aligned}
\bar{q}_{5}(X=0, \bar{Y}=0) & \leftarrow \bar{q}_{6}\left(\bar{u}_{5}=\bar{X}=0, \bar{v}_{5}=\frac{\bar{Y}}{\bar{X}}=0\right) \leftarrow \bar{q}_{7}\left(\bar{u}_{6}=\bar{u}_{5}=0, \bar{v}_{6}=\frac{\bar{v}_{5}}{\bar{u}_{5}}=\frac{c}{c-1}\right) \\
& \leftarrow \bar{q}_{8}\left(\bar{u}_{7}=\bar{u}_{6}=0, \bar{v}_{7}=\frac{(c-1) \bar{v}_{6}-c}{(c-1) \bar{u}_{6}}=\frac{c(c(\alpha+\beta+n+1)+n+1-\gamma)}{(c-1)^{2}}\right)
\end{aligned}
$$

for the degeneration cascade. 
and so $\left(\psi_{1}\right)_{*}\left(F_{5}-F_{6}\right)=\bar{F}_{7}-\bar{F}_{8}$. Note that the mappings $\left(\psi_{i}\right)_{*}$ should preserve the intersection form, and thus the self-intersection index. Indeed $\left(F_{5}-F_{6}\right)^{2}=\left(\bar{F}_{7}-\bar{F}_{8}\right)^{2}=-2$, as it should be. Other computations in the degeneration cascade are similar and result in

$$
\left(\psi_{1}\right)_{*}\left(F_{6}-F_{7}\right)=\bar{F}_{6}-\bar{F}_{7}, \quad\left(\psi_{1}\right)_{*}\left(F_{7}-F_{8}\right)=\bar{F}_{5}-\bar{F}_{6}, \quad\left(\psi_{1}\right)_{*}\left(F_{8}\right)=\bar{H}_{x}-\bar{F}_{5}
$$

Passing to classes, we get

$$
\left(\psi_{1}\right)_{*}\left(\mathcal{F}_{7}\right)=\left(\psi_{1}\right)_{*}\left(\mathcal{F}_{7}-\mathcal{F}_{8}\right)+\left(\psi_{1}\right)_{*}\left(\mathcal{F}_{8}\right)=\left(\overline{\mathcal{F}}_{5}-\overline{\mathcal{F}}_{6}\right)+\left(\overline{\mathcal{H}}_{x}-\overline{\mathcal{F}}_{5}\right)=\overline{\mathcal{H}}_{x}-\overline{\mathcal{F}}_{6},
$$

and so on.

To find $\left(\psi_{1}\right)_{*}\left(\mathcal{H}_{y}\right)$, it is convenient to choose a vertical line passing through some base points. For example $H_{y}-F_{5}-F_{6}$ is the proper transform of the line $y=\infty$, and we get

$$
(\bar{x}, \bar{y})(Y=0)=\left(x, x^{2}+\alpha \beta-x(n+1+\alpha+\beta)\right)
$$

which parameterizes the $(2,1)$-curve $\bar{\gamma}$ in the range given by the equation $\bar{x}^{2}+\alpha \beta-\bar{x}(n+1+\alpha+\beta)-\bar{y}=0$. Taking into account the evolution of parameters we note that this curve corresponds to the $(2,1)$-curve $\gamma$ in the domain given by

$$
\gamma: \quad x^{2}+\alpha \beta-x(n+\alpha+\beta)-y=0
$$

Since the self-intersection index of $\bar{\gamma}$ should be -2 , we expect it to pass through 6 (or fewer, in case of multiplicities) base points in the range. Indeed, we can check that it passes through $\bar{q}_{1}, \ldots, \bar{q}_{6}$, each with multiplicity one, and so

$\left(\psi_{1}\right)_{*}\left(\mathcal{H}_{y}\right)=\left(\psi_{1}\right)_{*}\left(\mathcal{H}_{y}-\mathcal{F}_{5}-\mathcal{F}_{6}\right)+\left(\psi_{1}\right)_{*}\left(\mathcal{F}_{5}\right)+\left(\psi_{1}\right)_{*}\left(\mathcal{F}_{6}\right)=\left(2 \overline{\mathcal{H}}_{x}+\overline{\mathcal{H}}_{y}-\bar{F}_{123456}\right)+\left(\overline{\mathcal{H}}_{x}-\overline{\mathcal{F}}_{8}\right)+\left(\overline{\mathcal{H}}_{x}-\bar{F}_{7}\right)$

This completes the computation for the forward mapping $\psi_{1}$. The computation for the backward mapping $\psi_{2}$ is similar, and the computation for the composed mapping $\psi^{(n)}$ immediately follows. 


\subsection{The Surface Type}

Given the base points $q_{1}, \ldots, q_{8}$, the point configuration is, essentially, the configuration of irreducible curves on which these points lie. These curves are in fact the irreducible components of some unique (unless we have very special values of parameters that correspond to an autonomous limit) bi-quadratic curve $\Gamma$. Let the equation of $\Gamma$ in the $(X, Y)$-chart be

$$
\left(a_{22} X^{2}+a_{12} X+a_{02}\right) Y^{2}+\left(a_{21} X^{2}+a_{11} X+a_{01}\right) Y+\left(a_{20} X^{2}+a_{10} X+a_{00}\right)=0 .
$$

From the condition $q_{5} \in \Gamma$ we see that $a_{00}=0$. To impose the condition that the infinitely close point (i.e., a point on an exceptional divisor) $q_{6} \in \Gamma$, we rewrite this equation in the $\left(u_{5}, v_{5}\right)$-chart (we should also include the $\left(U_{5}, V_{5}\right)$-chart, but unless it gives any new information, we omit those computations) via the substitution $X=u_{5}, Y=u_{5} v_{5}$. The resulting equation factorizes,

$$
u_{5}\left(\left(a_{22} u_{5}^{2}+a_{12} u_{5}+a_{02}\right) u_{5} v_{5}^{2}+\left(a_{21} u_{5}^{2}+a_{11} u_{5}+a_{01}\right) v_{5}+a_{20} u_{5}+a_{10}\right)=0 .
$$

This factorization corresponds to the decomposition of the total transform of $\Gamma$ under the blowup mapping $\mathrm{Bl}_{q_{5}}: X_{q_{5}} \rightarrow \mathbb{P}^{1} \times \mathbb{P}^{1}$ into the irreducible components, $\mathrm{Bl}_{q_{5}}^{-1}(\Gamma)=F_{5}+\left(\Gamma-F_{5}\right)$, where $F_{5}$ is the central fiber of the blowup, and $\Gamma-F_{5}$ is the proper transform of $\Gamma$. We then see that the condition $q_{6} \in \Gamma-F_{5}$ implies $a_{10}=0$. Continuing in this way through the degeneration cascade at $q_{5}$, as well as imposing the conditions $q_{i} \in \Gamma$ for $i=1, \ldots, 4$, we get the following equation for $\Gamma$ :

$$
\Gamma=V(Y s(X, Y)), \quad \text { where } \quad s(X, Y)=X^{2}-\alpha \beta X^{2} Y+(n+\alpha+\beta) X Y-Y .
$$


The curve $\Gamma$ is in fact the pole divisor of a symplectic form $\omega$, and that is why $\Gamma$ is called an anti-canonical divisor, $[\Gamma]=-\mathcal{K}_{x}$. Note also that the equation $s(X, Y)=0$ is nothing but the equation of the curve $\gamma$ in (2.5) written in the $(X, Y)$-chart. This is not surprising, since both $H_{y}-F_{5}-F_{6}$ and $\gamma$ are -2 -curves that are irreducible components of the anti-canonical divisor, and so they are permuted by the mapping.

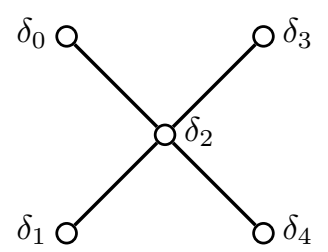

$$
\begin{aligned}
\delta_{0} & =\mathcal{F}_{5}-\mathcal{F}_{6}, \\
\delta_{1} & =2 \mathcal{H}_{x}+\mathcal{H}_{y}-\mathcal{F}_{123456}, \\
\delta_{2} & =\mathcal{F}_{6}-\mathcal{F}_{7},
\end{aligned}
$$

Figure 3: The Surface Root Basis for the Hypergeometric Weight Recurrence

This symplectic form $\omega$ in the affine $(X, Y)$-chart is given by

$$
\omega=k \frac{d X \wedge d Y}{s(X, Y) Y}=k \frac{d X \wedge d s}{s\left(s-X^{2}\right)}
$$

since

$$
d s=(2 X-2 \alpha \beta X Y+(n+\alpha+\beta) Y) d X-\left(\alpha \beta X^{2}-(n+\alpha+\beta) X+1\right) d Y
$$

and

$$
\frac{X^{2}-s(X, Y)}{Y}=\alpha \beta X^{2}-(n+\alpha+\beta) X+1 .
$$

This point configuration, the blowup diagram, and the decomposition of the anti-canonical divisor $-K_{x}=\Gamma$ is shown on Figure 2. Thus, we see that $-K_{x}$ decomposes into irreducible components as follows:

$$
-K_{x}=\left(2 H_{x}+H_{y}-F_{1}-F_{2}-F_{3}-F_{4}-F_{5}-F_{6}\right)+\left(H_{y}-F_{5}-F_{6}\right)+\left(F_{5}-F_{6}\right)+2\left(F_{6}-F_{7}\right)+\left(F_{7}-F_{8}\right),
$$

whose intersection structure is given by the $D_{4}^{(1)}$ affine Dynkin diagram shown in Figure 3 , where $\delta_{i}=\left[d_{i}\right]$.

Note that here the assignment of $d_{i}$ is arbitrary with the exception of $d_{2}$. Also, at this point we see that our equation is of type $\mathrm{d}-\mathrm{P}\left(\mathrm{D}_{4}^{(1)} / \mathrm{D}_{4}^{(1)}\right)$, and so our recurrence falls into the same family as the $\mathrm{d}-\mathrm{P}_{\mathrm{V}}$ equation. However, to see whether our recurrence is equivalent to $\mathrm{d}_{-} \mathrm{P}_{\mathrm{V}}$, we need to compare the dynamics. We describe the choice of the standard d-P $\left(D_{4}^{(1)} / D_{4}^{(1)}\right)$ point configuration, choices of the root bases for the surface and the symmetry sub-lattices, and other data, in the Appendix; we follow [KNY17] in our conventions.

\subsection{Initial Geometry Identification}

To compare the application dynamics with the standard dynamics of $\mathrm{d}-\mathrm{P}_{\mathrm{V}}$, we need to work with the same root bases. Thus, we begin by finding some change of basis of $\operatorname{Pic}(\mathcal{X})$ that will identify the surface roots between our recurrence and the standard example, and then use this change of basis to identify the symmetry roots and compare the translations. At this point, although we need to make some choices, we do not need to worry whether those choices are correct, since they will be adjusted later on.

Lemma 3. The following change of basis of $\operatorname{Pic}(X)$ identifies the root bases between the standard $D_{4}^{(1)}$ surface 
and the surface that we obtained for the hypergeometric weight recurrence:

$$
\begin{aligned}
\mathcal{H}_{x} & =\mathcal{H}_{g}, \\
\mathcal{H}_{y} & =\mathcal{H}_{f}+2 \mathcal{H}_{g}-\mathcal{E}_{3}-\mathcal{E}_{4}-\mathcal{E}_{5}-\mathcal{E}_{6}, \\
\mathcal{F}_{1} & =\mathcal{E}_{1}, \\
\mathcal{F}_{2} & =\mathcal{E}_{2}, \\
\mathcal{F}_{3} & =\mathcal{H}_{g}-\mathcal{E}_{6}, \\
\mathcal{F}_{4} & =\mathcal{H}_{g}-\mathcal{E}_{5}, \\
\mathcal{F}_{5} & =\mathcal{H}_{g}-\mathcal{E}_{4}, \\
\mathcal{F}_{6} & =\mathcal{H}_{g}-\mathcal{E}_{3}, \\
\mathcal{F}_{7} & =\mathcal{E}_{7}, \\
\mathcal{F}_{8} & =\mathcal{E}_{8},
\end{aligned}
$$

$$
\begin{aligned}
\mathcal{H}_{f} & =2 \mathcal{H}_{x}+\mathcal{H}_{y}-\mathcal{F}_{3}-\mathcal{F}_{4}-\mathcal{F}_{5}-\mathcal{F}_{6}, \\
\mathcal{H}_{g} & =\mathcal{H}_{x}, \\
\mathcal{E}_{1} & =\mathcal{F}_{1}, \\
\mathcal{E}_{2} & =\mathcal{F}_{2}, \\
\mathcal{E}_{3} & =\mathcal{H}_{x}-\mathcal{F}_{6}, \\
\mathcal{E}_{4} & =\mathcal{H}_{x}-\mathcal{F}_{5}, \\
\mathcal{E}_{5} & =\mathcal{H}_{x}-\mathcal{F}_{4}, \\
\mathcal{E}_{6} & =\mathcal{H}_{x}-\mathcal{F}_{3}, \\
\mathcal{E}_{7} & =\mathcal{F}_{7}, \\
\mathcal{E}_{8} & =\mathcal{F}_{8} .
\end{aligned}
$$

Proof. Consider the surface sub-lattice root basis on Figure 3 and compare it with the standard one on Figure 6. Since the $D_{4}^{(1)}$ affine Dynkin diagram has the distinguished node $\delta_{2}$, we must have

$$
\delta_{2}=\mathcal{F}_{6}-\mathcal{F}_{7}=\mathcal{H}_{g}-\mathcal{E}_{3}-\mathcal{E}_{7}
$$

Thus, we can put $\mathcal{F}_{6}=\mathcal{H}_{g}-\mathcal{E}_{3}$ and $\mathcal{F}_{7}=\mathcal{E}_{7}$, and then, matching $\mathcal{F}_{7}-\mathcal{F}_{8}=\mathcal{E}_{7}-\mathcal{E}_{8}$, we see that we can put $\mathcal{F}_{8}=\mathcal{E}_{8}$. Next, matching $\mathcal{F}_{5}-\mathcal{F}_{6}=\mathcal{E}_{3}-\mathcal{E}_{4}$, we see that $\mathcal{F}_{5}=\mathcal{H}_{g}-\mathcal{E}_{4}$. Matching $\mathcal{H}_{y}-\mathcal{F}_{5}-\mathcal{F}_{6}=\mathcal{H}_{f}-\mathcal{E}_{5}-\mathcal{E}_{6}$ we get $\mathcal{H}_{y}$. The final node matching gives us the equation $2 \mathcal{H}_{x}-\mathcal{F}_{1234}=\mathcal{E}_{5}+\mathcal{E}_{6}-\mathcal{E}_{1}-\mathcal{E}_{2}$. Thus, we can put (again, at this point we do not worry about making the right choice) $\mathcal{F}_{1}=\mathcal{E}_{1}, \mathcal{F}_{2}=\mathcal{E}_{2}, \mathcal{E}_{5}=\mathcal{H}_{x}-\mathcal{F}_{4}$ and $\mathcal{E}_{6}=\mathcal{H}_{x}-\mathcal{F}_{3}$, so that $\mathcal{H}_{x}=\mathcal{E}_{6}+\mathcal{F}_{3}=\mathcal{H}_{g}$. The inverse change of basis is straightforward.

\subsection{The Symmetry Roots and the Translations}

We are now in a position to compare the dynamics. Starting with the standard choice of the symmetry root basis (A.3) and using the change of basis in Lemma 3, we get the symmetry roots for the applied problem shown on Figure 4.

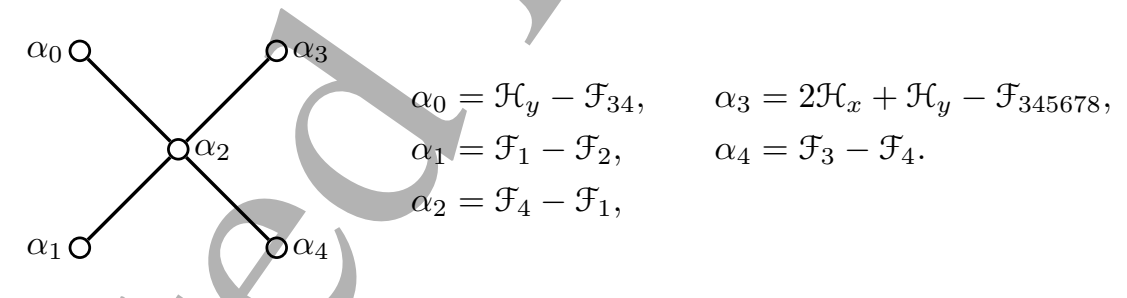

Figure 4: The Symmetry Root Basis for the Hypergeometric Weight Recurrence (preliminary choice)

From the action of $\psi_{*}$ on $\operatorname{Pic}(X)$ given in Lemma 2 we immediately see that the corresponding translation on the root lattice is

$$
\psi_{*}: \alpha=\left\langle\alpha_{0}, \alpha_{1}, \alpha_{2}, \alpha_{3}, \alpha_{4}\right\rangle \mapsto \psi_{*}(\alpha)=\alpha+\langle 1,0,0,-1,0\rangle \delta
$$

which is different from the standard translation vector $\langle 1,0,-1,1,0\rangle$ given in (A.20). However, decomposing $\psi$ in terms of generators of the extended affine Weyl symmetry group, see Section A.3, and comparing it with the expression for $\varphi$ given in (A.22),

$$
\psi=\sigma_{3} \sigma_{2} w_{3} w_{2} w_{4} w_{1} w_{2} w_{3}, \quad \varphi=\sigma_{3} \sigma_{2} w_{3} w_{0} w_{2} w_{4} w_{1} w_{2},
$$

we immediately see that $\psi=w_{3} \circ \varphi \circ w_{3}^{-1}$ (recall that $w_{3} \sigma_{3} \sigma_{2}=\sigma_{3} \sigma_{2} w_{0}$ and that $w_{3}$ is an involution, $\left.w_{3}^{-1}=w_{3}\right)$. Thus, our dynamic is indeed equivalent to the standard $\mathrm{d}-\mathrm{P}_{\mathrm{V}}$ equation, but the change of basis in Lemma 3 needs to be adjusted by acting by $w_{3}$. 
Remark 4. At this point we verified the decompositions in (2.10) on the level of the Picard lattice. That is, if we use expressions for symmetry roots in (2.9) to define $w_{i}$ and $\sigma_{i}$ as acting on the Picard lattice, we get the expression for the mapping $\left(\psi^{(n)}\right)_{*}$ in Lemma 2. To obtain this decomposition on the level of actual maps first requires finding the change of variables that induces the change of basis in Lemma 3 and then using it to rewrite the birational representation in Section A.3 in the application coordinates $(x, y)$. However, since the translation vectors do not match, we first adjust the linear change of basis on the Picard lattice,in Section 2.6 to match the dynamics, and only then find the change of coordinates for this adjusted/change of basis in Section 2.8.

\subsection{Final Geometry Identification}

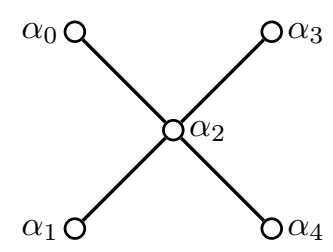

$$
\begin{aligned}
\alpha_{0} & =\mathcal{H}_{y}-\mathcal{F}_{34}, \\
\alpha_{1} & =\mathcal{F}_{1}-\mathcal{F}_{2}, \\
\alpha_{2} & =2 \mathcal{H}_{x}+\mathcal{H}_{y}-\mathcal{F}_{135678},
\end{aligned}
$$

Figure 5: The Symmetry Root Basis for the Hypergeometric Weight Recurrence (final choice)

Lemma 5. After the change of basis of $\operatorname{Pic}(X)$ given by

$$
\begin{array}{rlrl}
\mathcal{H}_{x} & =\mathcal{H}_{f}+\mathcal{H}_{g}-\mathcal{E}_{7}-\mathcal{E}_{8}, & \mathcal{H}_{f} & =2 \mathcal{H}_{x}+\mathcal{H}_{y}-\mathcal{F}_{3}-\mathcal{F}_{4}-\mathcal{F}_{5}-\mathcal{F}_{6}, \\
\mathcal{H}_{y} & =3 \mathcal{H}_{f}+2 \mathcal{H}_{g}-\mathcal{E}_{3}-\mathcal{E}_{4}-\mathcal{E}_{5}-\mathcal{E}_{6}-2 \mathcal{E}_{7}-2 \mathcal{E}_{8}, & \mathcal{H}_{g} & =3 \mathcal{H}_{x}+\mathcal{H}_{y}-\mathcal{F}_{3}-\mathcal{F}_{4}-\mathcal{F}_{5}-\mathcal{F}_{6}-\mathcal{F}_{7}-\mathcal{F}_{8}, \\
\mathcal{F}_{1} & =\mathcal{E}_{1}, & \mathcal{E}_{1} & =\mathcal{F}_{1}, \\
\mathcal{F}_{2} & =\mathcal{E}_{2}, & \mathcal{E}_{2} & =\mathcal{F}_{2}, \\
\mathcal{F}_{3} & =\mathcal{H}_{f}+\mathcal{H}_{g}-\mathcal{E}_{6}-\mathcal{E}_{7}-\mathcal{E}_{8}, & \mathcal{E}_{3} & =\mathcal{H}_{x}-\mathcal{F}_{6}, \\
\mathcal{F}_{4} & =\mathcal{H}_{f}+\mathcal{H}_{g}-\mathcal{E}_{5}-\mathcal{E}_{7}-\mathcal{E}_{8}, & \mathcal{E}_{4}=\mathcal{H}_{x}-\mathcal{F}_{5}, \\
\mathcal{F}_{5}=\mathcal{H}_{f}+\mathcal{H}_{g}-\mathcal{E}_{4}-\mathcal{E}_{7}-\mathcal{E}_{8}, & \mathcal{E}_{5}=\mathcal{H}_{x}-\mathcal{F}_{4}, \\
\mathcal{F}_{6}=\mathcal{H}_{f}+\mathcal{H}_{g}-\mathcal{E}_{3}-\mathcal{E}_{7}-\mathcal{E}_{8}, & \mathcal{E}_{6}=\mathcal{H}_{x}-\mathcal{F}_{3}, \\
\mathcal{F}_{7}=\mathcal{H}_{f}-\mathcal{E}_{8}, & \mathcal{E}_{7}=2 \mathcal{H}_{x}+\mathcal{H}_{y}-\mathcal{F}_{3}-\mathcal{F}_{4}-\mathcal{F}_{5}-\mathcal{F}_{6}-\mathcal{F}_{8}, \\
\mathcal{F}_{8}=\mathcal{H}_{f}-\mathcal{E}_{7}, & \mathcal{E}_{8}=2 \mathcal{H}_{x}+\mathcal{H}_{y}-\mathcal{F}_{3}-\mathcal{F}_{4}-\mathcal{F}_{5}-\mathcal{F}_{6}-\mathcal{F}_{7},
\end{array}
$$

the recurrence relations for variables $x_{n}$ and $y_{n}$ coincides with the standard $d-P_{\mathrm{V}}$ discrete Painleve equation given by (A.19). The resulting identification of the symmetry root bases (the surface root bases do not change) is shown in Figure 5.

Next we need to realize this change of basis on $\operatorname{Pic}(X)$ by an explicit change of coordinates. For that, it is convenient to first match the parameters between the applied problem and the reference example. This is done with the help of the Period Map.

\subsection{The Period Map and the Identification of Parameters}

The Period Map computation is similar to the slightly simpler standard case explained in Section A.2. Thus, we only state the result.

\section{Lemma 6.}


(i) The residue of the symplectic form $\omega=k \frac{d X \wedge d Y}{s(X, Y) Y}=k \frac{d X \wedge d s}{s\left(s-X^{2}\right)}$ defined in (2.7) along the irreducible components of the polar divisor is given by

$$
\begin{aligned}
\operatorname{res}_{d_{0}} \omega & =-k \frac{d v_{5}}{v_{5}^{2}}, & \operatorname{res}_{d_{2}} \omega & =-k \frac{(n+\alpha+\beta) d v_{6}}{\left(v_{6}-1\right)^{2}}, \quad \operatorname{res}_{d_{4}} \omega=-k \frac{d X}{X^{2}} \\
\operatorname{res}_{d_{1}} \omega & =k \frac{d X}{X^{2}}, & \operatorname{res}_{d_{3}} \omega & =-k \frac{(c-1)^{2} d v_{7}}{c} .
\end{aligned}
$$

(ii) The root variables are given by

$$
a_{0}=k(\gamma-n-\alpha), \quad a_{1}=k(\alpha-1), \quad a_{2}=k(1+n+\beta-\gamma), \quad a_{3}=-k(n+\beta), \quad a_{4}=k(\gamma-\beta) .
$$

The normalization condition $a_{0}+a_{1}+2 a_{2}+a_{3}+a_{4}=1$ then implies that $k=1$, and we get the following relations between our parameters and the root variables:

$$
\alpha=a_{1}+1, \quad \beta=a_{0}+a_{1}+a_{2}, \quad \gamma=1-a_{2}-a_{3}, \quad n=a_{2}+a_{4}-1 .
$$

Note that the root variable evolution, which is the same as given in (A.20), is consistent with what we expect: $\bar{\alpha}=\alpha, \bar{\beta}=\beta, \bar{\gamma}=\gamma$, and $\bar{n}=n+1$. Also observe that we can not yet see the relationship between parameters $t$ and $c$ in this identification. After we find the actual change of coordinates in the next section, we get that $c t=1$.

\subsection{The Change of Coordinates}

We are now ready to prove Theorem 1, which is the main result of the paper.

\section{Proof. (Theorem 1)}

Since equations (1.12) are simpler, we explain how to obtain them. Equations (1.11) can then be either obtained in the same way or by finding the explicit inverse change of variables from (1.12).

From our change of basis, we see that

$$
\mathcal{H}_{f}=2 \mathcal{H}_{x}+\mathcal{H}_{y}-\mathcal{F}_{3}-\mathcal{F}_{4}-\mathcal{F}_{5}-\mathcal{F}_{6}
$$

Thus, $f(x, y)$ is a projective coordinate on a pencil of $(2,1)$-curves in the $(x, y)$-plane passing through points $q_{3}, q_{4}, q_{5}$, and $q_{6}$. Working in the $(X, Y)$-chart, we consider a generic $(2,1)$-curve $a_{00}+a_{01} X+a_{02} X^{2}+$ $a_{10} Y+a_{11} X Y+a_{12} X^{2} Y=0$. To pass through $q_{5}(0,0)$ we much have $a_{00}=0$, and to pass through the point $q_{6}(X=0, Y / X=0)$ we must have $a_{10}=0$. Imposing conditions at $q_{3}$ and $q_{4}$ gives us more constraints on the coefficients, and we get

$$
a_{11}(Y(1-X \beta)(1-X \gamma))-a_{20}\left(Y(n+\alpha-\gamma)-X^{2}(\beta Y(\alpha \beta-\beta \gamma-n \gamma)-(\beta+\gamma))\right)=0 .
$$

The expressions at the coefficients $a_{11}$ and $a_{20}$ define two basis curves in the pencil and the coordinate $f(X, Y)$ is their ratio, up to a Möbius transformation. When written in the $(x, y)$-chart, we get

$$
f(x, y)=\frac{A(x-\beta)(x-\gamma)+B\left(x^{2}(n+\alpha-\gamma)+y(\beta+\gamma)-\beta(\alpha \beta-\beta \gamma-n \gamma)\right)}{C(x-\beta)(x-\gamma)+D\left(x^{2}(n+\alpha-\gamma)+y(\beta+\gamma)-\beta(\alpha \beta-\beta \gamma-n \gamma)\right)},
$$

where the coefficients $A, B, C, D$ are still to be determined. To do that, we use the information about the exceptional divisor correspondence in Lemma 5. For example, the condition $\mathcal{E}_{2}=\mathcal{F}_{2}$ means that $(f, g)\left(q_{2}\right)=(f, g)(\alpha,-n \alpha)=\left(\infty, b_{2}\right)=p_{2}$, i.e.,

$$
F(\alpha,-n \alpha)=\frac{C+D(n+\alpha+\beta)}{A+B(n+\alpha+\beta)}=0, \quad \text { and so } \quad C=-D(n+\alpha+\beta) .
$$


The condition $\mathcal{E}_{6}=\mathcal{H}_{x}-\mathcal{F}_{3}$ means that $f(\beta, y)=B / D=0$, and so $B=0$. As a result, after some simplifications, we get

$$
f(x, y)=\frac{\tilde{A}(x-\beta)(x-\gamma)}{(x-\alpha)(x-\beta)-n x-y},
$$

where $\tilde{A}$ is some proportionality constant. To find $\tilde{A}$, we use the condition $\mathcal{E}_{3}-\mathcal{E}_{4}=\mathcal{F}_{5}-\mathcal{F}_{6}$, which means that, after doing a sequence of substitutions to express $f$ in the $\left(u_{5}, v_{5}\right)$-chart and then restrictíng to $u_{5}=0$, the image of the (proper transform $\mathcal{F}_{5}-\mathcal{F}_{6}$ ) of the exceptional divisor $\mathcal{F}_{5}$ should collapse to the point $p_{3}(t, \infty)$, i.e., $f\left(u_{5}=0, v_{5}\right)=t$. This results in $A=1$. Similarly, the condition $\mathcal{E}_{7}-\mathcal{E}_{8}=\mathcal{F}_{7}-\mathcal{F}_{8}$ results in the relationship between $c$ and $t, c t=1$. Computing $g(x, y)$ is done exactly along the same lines, but the equations for the basis curves in the $\mathcal{H}_{g}$ pencil are more complicated, and so this computation is omitted.

\subsection{Partial Decompositions and Gauge Ambiguities}

In this section we want to make the following important point. Note that equation (1.5) is a relation between $x_{n}, y_{n}$, and $y_{n+1}$ that we used to define the forward map $\psi_{1}:\left(x_{n}, y_{n}\right) \rightarrow\left(x_{n}, y_{n+1}\right)$. Similarly, equation (1.6) is a relation between $x_{n-1}, x_{n}$, and $y_{n}$ that we used to define the backward map $\psi_{2}:\left(x_{n}, y_{n}\right) \rightarrow\left(x_{n-1}, y_{n}\right)$. In doing so we ignored possible $\mathbf{P G L}_{2}(\mathbb{C}) \times \mathbf{P} \mathbf{G L}_{2}(\mathbb{C})$ gauge group actions on both the domain and the range of the mappings. Thus, the mappings $\psi_{i}$ may not correspond exactly to elements of the birational representation of the symmetry group, where some normalization must be imposed to ensure the group structure on the level of the mappings. This point is essential, since we may not see the correct evolution of parameters in these partial maps. If necessary, this problem can be corrected using the action of the mappings on the Picard lattice (that does not depend on the gauge actions) and the Period Map.

This issue can already be seen in the simpler model example of the difference Painlevé-V equation (A.19). This mapping can also be partially decomposed, in the natural way, as $\varphi=\varphi_{2}^{-1} \circ \varphi_{1}$, where $\varphi_{1}$ is a forward mapping $\varphi_{1}:(f, g) \mapsto(\bar{f},-g)$ and $\varphi_{2}$ is a backward mapping $\varphi_{2}:(f, g) \mapsto(f,-g)$. Note that the additional negative sign (which is an example of the gauge group action mentioned above) is essential for the mappings $\varphi_{i}$ to be representable as a composition of elementary birational maps described in Theorems 10 and 11 , where the normalization condition that we imposed in constructing the birational representation of $\widetilde{W}\left(D_{4}^{(1)}\right)$ is given by (A.9). In fact, there are two slightly different ways to write these mappings in terms of generators; $\varphi=\varphi_{2}^{-1} \circ \varphi_{1}=\tilde{\varphi}_{2}^{-1} \circ \tilde{\varphi}_{1}$ (this is a direct calculation):

$$
\begin{aligned}
& \varphi_{1}=\sigma_{3} \sigma_{2} w_{1} w_{2} w_{4} w_{1} w_{2}:(f, g) \mapsto(\bar{f},-g) ; \quad \bar{a}_{0}=1-a_{0}, \bar{a}_{1}=a_{1}, \bar{a}_{2}=-a_{1}-a_{2}, \bar{a}_{3}=1-a_{3}, \bar{a}_{4}=-a_{4} ; \\
& \varphi_{2}=w_{0} w_{3} w_{4}:(f, g) \mapsto(f,-\underline{g}) ; \quad \underline{a}_{0}=-a_{0}, \underline{a}_{1}=a_{1}, \underline{a}_{2}=1-a_{1}-a_{2}, \underline{a}_{3}=-a_{3}, \underline{a}_{4}=-a_{4}, \quad(2.16)
\end{aligned}
$$

Or

$$
\begin{aligned}
& \tilde{\varphi}_{1}=\sigma_{3} \sigma_{2} w_{1} w_{2} w_{4} w_{1} w_{2} w_{1}:(f, g) \mapsto(\bar{f},-g) ; \quad \bar{a}_{0}=1-a_{0}, \bar{a}_{1}=-a_{1}, \bar{a}_{2}=-a_{2}, \bar{a}_{3}=1-a_{3}, \bar{a}_{4}=-a_{4} \\
& \tilde{\varphi}_{2}=w_{0} w_{1} w_{3} w_{4}:(f, g) \mapsto(f,-\underline{g}) ; \quad \underline{a}_{0}=-a_{0}, \underline{a}_{1}=-a_{1}, \underline{a}_{2}=1-a_{2}, \underline{a}_{3}=-a_{3}, \underline{a}_{4}=-a_{4} .
\end{aligned}
$$

Looking at the action of the mappings on the root variables $a_{i}$ it is clear that the individual mappings $\varphi_{1,2}$ do not correspond to translations on the symmetry sub-lattice; in fact, the need for the negative sign can be clearly seen at this point. The nêgative sign disappears, which is fairly typical, when we consider complete forward or backward steps in the dynamics, since those correspond to translations

$$
(\underline{f}, \underline{g}) \stackrel{\varphi_{1}^{-1}}{\longleftarrow}(f,-\underline{g}) \stackrel{\varphi_{2}}{\longleftarrow}(f, g) \stackrel{\varphi_{1}}{\longrightarrow}(\bar{f},-g) \stackrel{\varphi_{2}^{-1}}{\longrightarrow}(\bar{f}, \bar{g}) \text {. }
$$

The same is true for the mappings $\psi_{i}$ from Lemma 2. Looking at the action of $\left(\psi_{1}\right)_{*}$ on the symmetry roots $(2.12)$, we get

$\left(\psi_{1}\right)_{*}\left(\alpha_{0}\right)=\delta-\alpha_{0},\left(\psi_{1}\right)_{*}\left(\alpha_{1}\right)=-\alpha_{1},\left(\psi_{1}\right)_{*}\left(\alpha_{2}\right)=\delta-\alpha_{2},\left(\psi_{1}\right)_{*}\left(\alpha_{3}\right)=-\delta-\alpha_{3},\left(\psi_{1}\right)_{*}\left(\alpha_{4}\right)=-\alpha_{4}$. 
This immediately gives us the decomposition of $\psi_{1}$ and the action on the root variables:

$$
\psi_{1}=\sigma_{3} \sigma_{2} w_{0} w_{1} w_{2} w_{4} w_{1} w_{2} w_{3} w_{1} ; \quad \bar{a}_{0}=1-a_{0}, \bar{a}_{1}=-a_{1}, \bar{a}_{2}=1-a_{2}, \bar{a}_{3}=-1-a_{3}, \bar{a}_{4}=-a_{4} \text {. (2.18) }
$$

Using (2.14) we get the evolution of parameters (that is non-physical, since weight parameters should not change)

$$
\bar{\alpha}=2-\alpha, \quad \bar{\beta}=2-\beta, \quad \bar{\gamma}=2-\gamma, \quad \bar{n}=-(n+1),
$$

which, in turn, gives us the forward evolution of the base points, which is different from the evolution given in Lemma 2(a):

$$
\begin{array}{ll}
\bar{q}_{1}(1,(\alpha-1)(\beta-1)+(n+1)), & \bar{q}_{2}(2-\alpha,(n+1)(2-\alpha)), \\
\bar{q}_{3}(2-\beta,(n+1)(2-\beta)), & \bar{q}_{4}(2-\gamma,(\alpha-\gamma)(\beta-\gamma)+(n+1)(2-\gamma)), \\
\bar{q}_{5}(\bar{X}=0, \bar{Y}=0) \leftarrow \bar{q}_{6}\left(\bar{u}_{5}=\bar{X}=0, \bar{v}_{5}=\frac{\bar{Y}}{\bar{X}}=0\right) \leftarrow \bar{q}_{7}\left(\bar{u}_{6}=\bar{u}_{5}=0, \bar{v}_{6}=\frac{\bar{v}_{5}}{\bar{u}_{5}}=\frac{c}{c-1}\right) \\
\leftarrow \bar{q}_{8}\left(\bar{u}_{7}=\bar{u}_{6}=0, \bar{v}_{7}=\frac{(c-1) \bar{v}_{6}-c}{(c-1) \bar{u}_{6}}=\frac{c(c(3-\alpha-\beta-n)-3-n+\gamma)}{(c-1)^{2}}\right) .
\end{array}
$$

Then the correct choice of the gauge to ensure that the mapping $\psi_{1}$ comes from the birational representation of the symmetry group is given by $\psi_{1}(x, y)=(2-x, \bar{y}+2(n+1))$, where $\bar{y}$ is given by $(2.1)$. This can either be deduced from the evolution of the base points (2.18) or obtained directly from the birational representation of $\psi_{1}$ using the change of variables (1.11-1.12).

Similarly, for $\left(\psi_{2}\right)_{*}$ the action on the symmetry roots is

$$
\left(\psi_{1}\right)_{*}\left(\alpha_{0}\right)=-\alpha_{0},\left(\psi_{1}\right)_{*}\left(\alpha_{1}\right)=-\alpha_{1},\left(\psi_{1}\right)_{*}\left(\alpha_{2}\right)=2 \delta-\alpha_{2},\left(\psi_{1}\right)_{*}\left(\alpha_{3}\right)=-2 \delta-\alpha_{3},\left(\psi_{1}\right)_{*}\left(\alpha_{4}\right)=-\alpha_{4} \text {. }
$$

The resulting decomposition of $\psi_{2}$ and the action on the root variables is $\psi_{2}=w_{4} w_{3} w_{2} w_{1} w_{0} w_{2} w_{4} w_{2} w_{1} w_{0} w_{2} w_{3} w_{1} w_{0} ; \quad \underline{a}_{0}=-a_{0}, \underline{a}_{1}=-a_{1}, \underline{a}_{2}=2-a_{2}, \underline{a}_{3}=-2-a_{3}, \underline{a}_{4}=-a_{4}$.

Using (2.14) we get the evolution of parameters (that is again non-physical)

$$
\underline{\alpha}=2-\alpha, \quad \underline{\beta}=2-\beta, \quad \underline{\gamma}=2-\gamma, \quad \underline{n}=-n,
$$

which, in turn, gives us the backward evolution of the base points, which is again different from the evolution given in Lemma 2(b):

$$
\begin{aligned}
& \underline{q}_{1}(1,(\alpha-1)(\beta-1)+n), \quad \underline{q}_{2}(2-\alpha, n(2-\alpha)), \\
& \underline{q}_{3}(2-\beta, n(2-\beta)), \quad \underline{q}_{4}(2-\gamma,(\alpha-\gamma)(\beta-\gamma)+n(2-\gamma)), \\
& \underline{q}_{5}(\underline{X}=0, \underline{Y}=0) \leftarrow \underline{q}_{6}\left(\underline{u}_{5}=\underline{X}=0, \underline{v}_{5}=\frac{\underline{Y}}{\underline{X}}=0\right) \leftarrow \underline{q}_{7}\left(\underline{u}_{6}=\underline{u}_{5}=0, \underline{v}_{6}=\frac{\underline{v}_{5}}{\underline{u}_{5}}=\frac{c}{c-1}\right) \\
& \leftarrow \underline{q}_{8}\left(\underline{u}_{7}=\underline{u}_{6}=0, \underline{v}_{7}=\frac{(c-1) \underline{v}_{6}-c}{(c-1) \underline{u}_{6}}=\frac{c(c(4-\alpha-\beta-n)-2-n+\gamma)}{(c-1)^{2}}\right) .
\end{aligned}
$$

Hence the correct choice of the gauge to ensure that the mapping $\psi_{2}$ comes from the birational representation of the symmetry group is given by $\psi_{2}(x, y)=(2-\underline{x}, y+2 n)$, where $\underline{x}$ is given by $(2.3)$.

Note that these gauge transformations cancel each other when we consider the full step. Indeed, let us define $\psi_{1}^{(n)}\left(x_{n}, y_{n}\right)=\left(2-x_{n}, y_{n+1}+2(n+1)\right)$ and $\psi_{2}^{(n)}\left(x_{n}, y_{n}\right)=\left(2-x_{n-1}, y_{n}+n\right)$. Then

$$
\psi^{(n)}\left(x_{n}, y_{n}\right)=\left(\psi_{2}^{(n+1)}\right)^{-1} \circ \psi_{1}^{(n)}\left(x_{n}, y_{n}\right)=\left(\psi_{2}^{(n+1)}\right)^{-1}\left(2-x_{n}, y_{n+1}+2(n+1)\right)=\left(x_{n+1}, y_{n+1}\right) \text {. }
$$

Remark 7. Given that both mappings $\psi$ and $\varphi$ decompose in a natural way, and that both mappings are equivalent, it is reasonable to ask whether these decompositions are equivalent individually. This, unfortunately, is not the case. Indeed, as can be seen from the above decompositions, $\psi_{1}=w_{3} \circ \varphi_{1} \circ w_{3}^{-1}$, but $\psi_{2}=\psi_{1} \circ \varphi^{-1}$ and this can not really be simplified much further. 


\section{Conclusions}

In this paper we illustrated a systematic procedure on determining whether a second-order non-linear nonautonomous recurrence relation is a discrete Painlevé equation, and if so, how to reduce it to the standard form. We considered in detail an example from the theory of discrete orthogonal polynomials, where we showed that the evolution of recurrence coefficients for these polynomials is expressed in terms of a particular solution of the standard difference Painlevé-V equation. However, it is clear that this approach can be easily adapted to a wide range of other applied problems where discrete Painlevé equations appear.

\section{A Standard example of d-P $\left(D_{4}^{(1)} / D_{4}^{(1)}\right)$}

In this section we review the standard example of discrete Painlevé equation of type d-P $\left(D_{4}^{(1)} / D_{4}^{(1)}\right)$, also known as the $\mathrm{d}-\mathrm{P}_{\mathrm{V}}$ equation. Note that this equation describes Bäcklund transformations of the usual differential $\mathrm{P}_{\mathrm{VI}}$ equation. We follow the standard reference [KNY17] for the choice of root bases and the form of the equation.

\section{A.1 The Point Configuration}

We start with the root basis of the surface sub-lattice that is given by the classes $\delta_{i}=\left[d_{i}\right]$ of the irreducible components of the anti-canonical divisor

$$
\delta=-\mathcal{K}_{x}=2 \mathcal{H}_{f}+2 \mathcal{H}_{g}-\mathcal{E}_{1}-\mathcal{E}_{2}-\mathcal{E}_{3}-\mathcal{E}_{4}-\mathcal{E}_{5}-\mathcal{E}_{6}-\mathcal{E}_{7}-\mathcal{E}_{8}=\delta_{0}+\delta_{1}+2 \delta_{2}+\delta_{3}+\delta_{4} .
$$

The intersection configuration of those roots is given by the Dynkin diagram of type $D_{4}^{(1)}$, as shown on Figure 6.

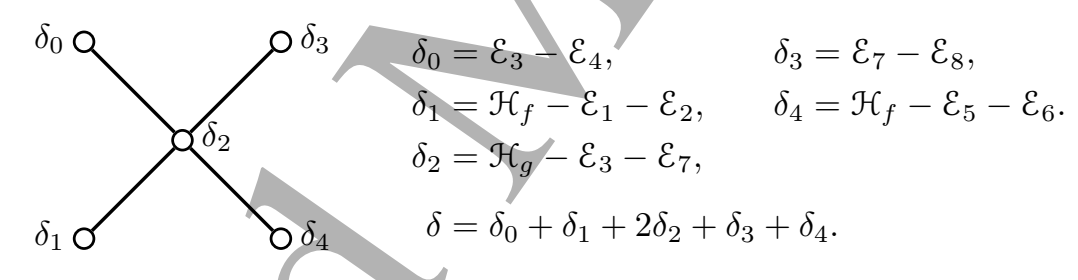

Figure 6: The Surface Root Basis for the standard d-P $\left(\mathrm{D}_{4}^{(1)}\right)$ point configuration

Using the action of the $\mathbf{P G L}_{2}(\mathbb{C}) \times \mathbf{P G L}_{2}(\mathbb{C})$ gauge group (i.e., the action of a Möbius group on each of the factors of $\left.\mathbb{P}^{1} \times \mathbb{P}^{1}\right)$, we can, without loss of generality, put $d_{i}$, with $\delta_{i}=\left[d_{i}\right]$ to be

$$
d_{1}=V(F)=\{f=\infty\}, \quad d_{2}=V(G)=\{g=\infty\}, \quad d_{4}=V(f)=\{f=0\},
$$

which then reduces the gauge group action to that of a three-parameter subgroup, $(f, g) \mapsto(\lambda f, \mu g+\nu)$. The corresponding point configuration and the Sakai surface are shown on Figure 7.

This point configuration can be parameterized by eight parameters $b_{1}, \ldots, b_{8}$ as follows:

$$
\begin{array}{lll}
p_{1}\left(\infty, b_{1}\right), & p_{2}\left(\infty, b_{2}\right), & p_{3}\left(b_{3}, \infty\right) \leftarrow p_{4}\left(b_{3}, \infty ; g\left(f-b_{3}\right)=b_{4}\right), \\
p_{5}\left(0, b_{5}\right), & p_{6}\left(0, b_{6}\right), & p_{7}\left(b_{7}, \infty\right) \leftarrow p_{8}\left(b_{7}, \infty ; g\left(f-b_{7}\right)=b_{8}\right) .
\end{array}
$$

The three-parameter gauge group above acts on these configurations via

$$
\left(\begin{array}{cccc}
b_{1} & b_{2} & b_{3} & b_{4} ; \\
b_{5} & b_{6} & b_{7} & b_{8} ; g
\end{array}\right) \sim\left(\begin{array}{llll}
\mu b_{1}+\nu & \mu b_{2}+\nu & \lambda b_{3} & \lambda \mu b_{4} \\
\mu b_{5}+\nu & \mu b_{6}+\nu & \lambda b_{7} & \lambda \mu b_{8} \\
\mu g & \lambda f+\nu
\end{array}\right), \lambda, \mu \neq 0
$$

and so the true number of parameters is five. The correct gauge-invariant parameterization is given by the root variables that we now describe. 


\section{A.2 The Period Map and the Root Variables}

To define the root variables we begin by choosing a root basis in the symmetry sub-lattice $Q=\Pi\left(R^{\perp}\right) \triangleleft \operatorname{Pic}(X)$ and defining the symplectic form $\omega$ whose polar divisor $-K_{x}$ is the configuration of -2 -curves shown on Figure 7. For the symmetry root basis we take the same basis as in [KNY17], see Figure 8.

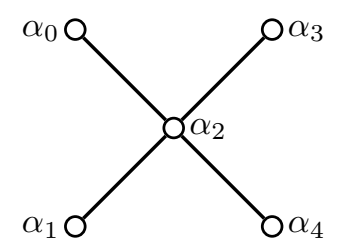

$$
\begin{aligned}
& \alpha_{0}=\mathcal{H}_{f}-\mathcal{E}_{3}-\mathcal{E}_{4}, \quad \alpha_{3}=\mathcal{H}_{f}-\mathcal{E}_{7}-\mathcal{E}_{8}, \\
& \alpha_{1}=\mathcal{E}_{1}-\mathcal{E}_{2}, \quad \alpha_{4}=\mathcal{E}_{5}-\mathcal{E}_{6} \text {. } \\
& \alpha_{2}=\mathcal{H}_{g}-\mathcal{E}_{1}-\mathcal{E}_{5} \text {, } \\
& \delta=\alpha_{0}+\alpha_{1}+2 \alpha_{2}+\alpha_{3}+\alpha_{4} .
\end{aligned}
$$

Figure 8: The Symmetry Root Basis for the standard d- $P\left(D_{4}^{(1)}\right)$ case

A symplectic form $\omega \in-\mathcal{K}_{x}$ such that $[\omega]=\delta_{0}+\delta_{1}+2 \delta_{2}+\delta_{3}+\delta_{4}$ can be given in local coordinate charts as

$$
\omega=k \frac{d f \wedge d g}{f}=-k \frac{d F \wedge d g}{F}=-k \frac{d f \wedge d G}{f G^{2}}=k \frac{d F \wedge d G}{F G^{2}}=-k \frac{d U_{3} \wedge d V_{3}}{\left(b_{3}+U_{3} V_{3}\right) V_{3}}=-k \frac{d U_{7} \wedge d V_{7}}{\left(b_{7}+U_{7} V_{7}\right) V_{7}},
$$

where, as usual, $F=1 / f, G=1 / g$ are the coordinates centered at infinity, the blowup coordinates $\left(U_{i}, V_{i}\right)$ at the points $p_{i}, i=3,7$, are given by $f=b_{i}+U_{i} V_{i}$ and $G=V_{i}$, and $k$ is some non-zero proportionality constant that we normalize later. Then we have the following Lemma.

\section{Lemma 8.}

(i) The residue of the symplectic form $\omega$ along the irreducible components of the polar divisor is given by

$$
\operatorname{res}_{d_{0}} \omega=k \frac{d U_{3}}{b_{3}}, \quad \operatorname{res}_{d_{1}} \omega=-k d g, \quad \operatorname{res}_{d_{2}} \omega=0, \quad \operatorname{res}_{d_{3}} \omega=k \frac{d U_{7}}{b_{7}}, \quad \operatorname{res}_{d_{4}} \omega=k d g .
$$

(ii) The root variables $a_{i}$ are given by

$$
a_{0}=-k \frac{b_{4}}{b_{3}}, \quad a_{1}=k\left(b_{2}-b_{1}\right), \quad a_{2}=k\left(b_{1}-b_{5}\right), \quad a_{3}=-k \frac{b_{8}}{b_{7}}, \quad a_{4}=k\left(b_{5}-b_{6}\right)
$$

It is convenient to take $k=-1$. We can then use the gauge action (A.2) to normalize $b_{5}=0, b_{7}=1$, and $\chi(\delta)=a_{0}+a_{1}+2 a_{2}+a_{3}+a_{4}=1$. In view of the relation of this example to $P_{\mathrm{VI}}$, it is also 
convenient to denote $b_{3}$ by $t$. Then we get the following parameterization of the point configuration in terms of root variables:

$$
b_{1}=-a_{2}, \quad b_{2}=-a_{1}-a_{2}, \quad b_{3}=t, \quad b_{4}=t a_{0}, \quad b_{5}=0, \quad b_{6}=a_{4}, \quad b_{7}=1, \quad b_{8}=a_{3} .
$$

Note that if we use the notation $p_{34}\left(t\left(1+\varepsilon a_{0}\right), 1 / \varepsilon\right), p_{78}\left(1+\varepsilon a_{3}, 1 / \varepsilon\right)$ for the degeneration cascades, we get exactly the parameterization of the point configuration in section 8.2.17 of [KNY17].

Proof. Part (a) is a standard computation in local charts. For example, with $d_{0}=E_{3}-E_{4}=V\left(V_{3}\right)$ in the chart $\left(U_{3}, V_{3}\right)$, we get

$$
\operatorname{res}_{d_{0}} \omega=\operatorname{res}_{V_{3}=0}\left(-k \frac{d U_{3} \wedge d V_{3}}{\left(b_{3}+U_{3} V_{3}\right) V_{3}}\right)=k \frac{d U_{3}}{b_{3}} .
$$

Other computations in part (a) are similar.

For part (b), first recall that the Period Map $\chi: Q \rightarrow \mathbb{C}$ is defined on the simple roots $\alpha_{i}$, where $a_{i}:=\chi\left(\alpha_{i}\right)$ are called the root variables, and then extended to the full symmetry sub-lattice by linearity. To compute the root variables $a_{i}$, we proceed as follows, see [Sak01] for details.

- First, we represent $\alpha_{i}$ as a difference of two effective divisors, $\alpha_{i}=\left[C_{i}^{1}\right]-\left[C_{1}^{0}\right]$;

- second, note that there exists a unique component $d_{k}$ of $-K_{x}$ such that $d_{k} \bullet C_{i}^{1}=d_{k} \bullet C_{i}^{0}=1$, put $P_{i}=d_{k} \cap C_{i}^{0}$ and $Q_{i}=d_{k} \cap C_{i}^{1}$ :

- then

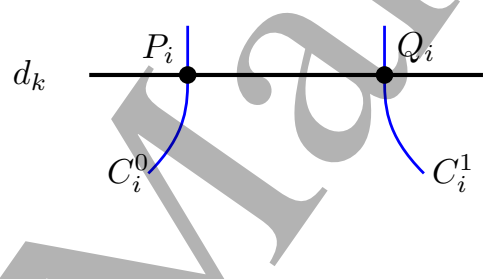

$$
\chi\left(\alpha_{i}\right)=\chi\left(\left[C_{i}^{1}\right]-\left[C_{i}^{0}\right]\right)=\int_{P_{i}}^{Q_{i}} \frac{1}{2 \pi \mathfrak{i}} \oint_{d_{k}} \omega=\int_{P_{i}}^{Q_{i}} \operatorname{res}_{d_{k}} \omega,
$$

where $\omega$ is the symplectic form defined by (A.4).

We illustrate this procedure by computing the root variable $a_{0}$, the other computations are similar (see also [DT18] for more examples of such computations). First represent $\alpha_{0}=\mathcal{H}_{f}-\mathcal{E}_{3}-\mathcal{E}_{4}=\left[H_{f}-E_{3}\right]-\left[E_{4}\right]$. These two curves intersect with the $d_{0}$ component of $\operatorname{div}(\omega)$, and so we get

$$
a_{0}=\chi\left(\alpha_{0}\right)=\int_{P_{0}}^{Q_{0}} \operatorname{res}_{d_{0}} \omega=k \int_{b_{4}}^{0} \frac{d U_{3}}{b_{3}}=-k \frac{b_{4}}{b_{3}}
$$




\section{A.3 The Extended Affine Weyl Symmetry Group}

For completeness, we also include here the description of the birational representation of the extended affine Weyl symmetry group $\widetilde{W}\left(D_{4}^{(1)}\right)=\operatorname{Aut}\left(D_{4}^{(1)}\right) \ltimes W\left(D_{4}^{(1)}\right)$, which is a semi-direct product of the usual affine Weyl group $W\left(D_{4}^{(1)}\right)$ and the group of Dynkin diagram automorphisms Aut $\left(D_{4}^{(1)}\right)$.

The affine Weyl group $W\left(D_{4}^{(1)}\right)$ is defined in terms of generators $w_{i}=w_{\alpha_{i}}$ and relations that are encoded by the affine Dynkin diagram $D_{4}^{(1)}$,

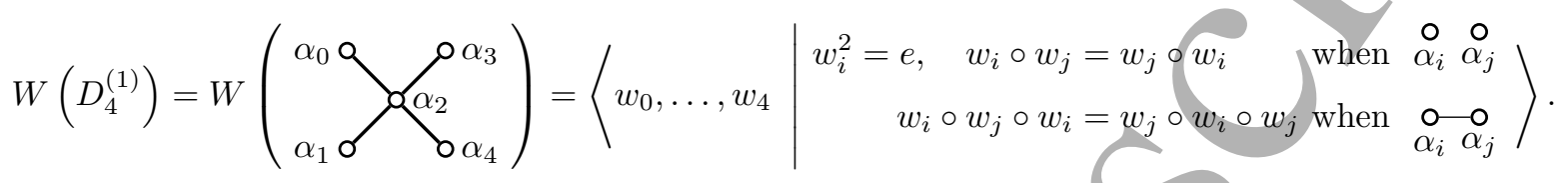

The natural action of this group on $\operatorname{Pic}(X)$ is given by reflections in the roots $\alpha_{i}$,

$$
w_{i}(\mathcal{C})=w_{\alpha_{i}}(\mathcal{C})=\mathcal{C}-2 \frac{\mathcal{C} \bullet \alpha_{i}}{\alpha_{i} \bullet \alpha_{i}} \alpha_{i}=\mathcal{C}+\left(\mathcal{C} \bullet \alpha_{i}\right) \alpha_{i}, \quad \mathcal{C} \in \operatorname{Pic}(X)
$$

which can be extended to an action on point configurations by elementary birational maps (which lifts to isomorphisms $w_{i}: X_{\mathbf{b}} \rightarrow X_{\overline{\mathbf{b}}}$ on the family of Sakai's surfaces), this is known as a birational representation of $W\left(D_{4}^{(1)}\right)$.

Remark 9. Recall that for an arbitrary $w \in \widetilde{W}\left(D_{4}^{(1)}\right)$, the action of $w$ on the root variables is inverse to its action on the roots. This is not essential for the generating reflections, that are involutions, but it is important for composed maps.

Theorem 10. Reflections $w_{i}$ on $\operatorname{Pic}(X)$ are induced by the elementary birational mappings given below and also denoted by $w_{i}$, on the family $X_{\mathbf{b}}$. To ensure the group structure, we require that each map preserves our normalization

$$
\left(\begin{array}{llll}
b_{1} & b_{2} & b_{3} & b_{4} \\
b_{5} & b_{6} & b_{7} & b_{8}
\end{array}\right)=\left(\begin{array}{cccc}
b_{1} & b_{2} & t & b_{4} \\
0 & b_{6} & 1 & b_{8}
\end{array}\right)=\left(\begin{array}{cccc}
-a_{2} & -a_{1}-a_{2} & t & t a_{0} \\
0 & a_{4} & 1 & a_{3}
\end{array}\right)
$$

We give the action of the mappings both on parameters $b_{i}$ related to the parameterization of point configurations, and on the root variables (note that the parameter $t$ can also change when we consider the Dynkin diagram automorphisms, so it is convenient to include it among the root variables). For the initial configuration

$$
\left(\begin{array}{ccccc}
b_{1} & b_{2} & t & b_{4} \\
0 & b_{6} & 1 & b_{8}
\end{array} ; g\right)=\left(\begin{array}{cccc}
a_{0} & a_{1} & a_{2} & f \\
a_{3} & a_{4} & t
\end{array} ; g\right)
$$

the action of $w_{i}$ is given by the following expressions: 
Proof. The proof is standard, see [DT18] for careful explanations, but to make this paper self-contained, we briefly outline one such computation. The reflection $w_{0}$ in the root $\alpha_{0}=\mathcal{H}_{f}-\mathcal{E}_{3}-\mathcal{E}_{4}$ acts on $\operatorname{Pic}(\mathcal{X})$ by $w_{0}\left(\mathcal{H}_{f}\right)=\mathcal{H}_{f}, w_{0}\left(\mathcal{H}_{g}\right)=\mathcal{H}_{f}+\mathcal{H}_{g}-\mathcal{E}_{3}-\mathcal{E}_{4}, w_{0}\left(\mathcal{E}_{3}\right)=\mathcal{H}_{f}-\mathcal{E}_{4}, w_{0}\left(\mathcal{E}_{4}\right)=\mathcal{H}_{f}-\mathcal{E}_{3}, w_{0}\left(\mathcal{E}_{i}\right)=\mathcal{E}_{i}, i \neq 3,4$. Thus, we are looking for a mapping $w_{0}: X_{\mathbf{b}} \rightarrow X_{\overline{\mathbf{b}}}$ that is given in the affine chart $(f, g)$ by a formula $w_{0}(f, g)=(\bar{f}, \bar{g})$ so that

$$
w_{0}^{*}\left(\mathcal{H}_{\bar{f}}\right)=\mathcal{H}_{f}, \quad w_{0}^{*}\left(\mathcal{H}_{\bar{g}}\right)=\mathcal{H}_{f}+\mathcal{H}_{g}-\mathcal{E}_{3}-\mathcal{E}_{4} .
$$

Thus, up to Möbius transformations, $\bar{f}$ coincides with $f$ and $\bar{g}$ is a coordinate on a pencil of $(1,1)$-curves passing through the degeneration cascade $p_{3}\left(b_{3}, \infty\right) \leftarrow p_{4}\left(b_{3}, \infty ; g\left(f-b_{3}\right)=b_{4}\right)$. Let $\left|\hat{H}_{\bar{g}}\right|=\{A f g+B f+$ $C g+D=0\}$. Then $p_{3}$ imposes the condition $A b_{3}+C=0$, and so $\left|H_{\bar{g}}\right|=\left\{A\left(f-b_{3}\right) g+B f+D=0\right\}$. Point $p_{4}$ then imposes the condition $A b_{4}+B b_{3}+D=0$ and we see that the basis of the pencil $\left|H_{\bar{g}}\right|$ is given by $\left(f-b_{3}\right) g-b_{4}$ and $f-b_{3}$. Taking the Möbius transformations into account, we get

$$
\bar{f}=\frac{A f+B}{C f+D}, \quad \bar{g}=\frac{K\left(\left(f-b_{3}\right) g-b_{4}\right)+L\left(f-b_{3}\right)}{M\left(\left(f-b_{3}\right) g-b_{4}\right)+N\left(f-b_{3}\right)^{\prime}},
$$

where $A, \ldots, N$ are some constants to be determined. We also know that the root variables change as $\bar{a}_{0}=-a_{0}, \bar{a}_{2}=a_{0}+a_{2}$, and $\bar{a}_{i}=a_{i}$ otherwise. This then gives us the evolution of parameters $b_{i}$, e.g., $\bar{b}_{1}=-\bar{a}_{2}=-a_{2}-a_{0}=b_{1}-b_{4} / t$ (recall that $t=b_{3}$ ), and so on. The constants $A, \ldots, N$ can be determined from the action of $w_{0}$ on exceptional divisors. For example, $w_{0}\left(\overline{\mathcal{E}}_{5}\right)=\mathcal{E}_{5}$ is equivalent to

$$
\begin{aligned}
& \qquad(\bar{f}, \bar{g})(0,0)=(0,0) \quad \Longrightarrow B=0, L=-K \frac{b_{4}}{b_{3}}, \\
& w_{0}\left(\bar{\varepsilon}_{7}\right)=\mathcal{E}_{7} \text { implies that } M=0 \text {, then } w_{0}\left(\overline{\mathcal{E}}_{1}\right)=\varepsilon_{1} \text { gives } \\
& \qquad(\bar{f}, \bar{g})\left(\infty, b_{1}\right)=\left(\infty, \bar{b}_{1}\right)=\left(\infty, b_{1}-b_{4} / t\right) \Longrightarrow C=0, \quad K / N=1, \\
& \text { and so on. }
\end{aligned}
$$

Let us now describe the group/of Dynkin diagram automorphisms. It is clear that $\operatorname{Aut}\left(D_{4}^{(1)}\right) \simeq \mathfrak{S}_{4}$, so we only describe three transpositions that generate the whole group.

Theorem 11. Consider the following generators $\sigma_{1}, \ldots, \sigma_{3}$ of Aut $\left(D_{4}^{(1)}\right)$ that act on the symmetry and the surface root bases as follows (here we use the standard cycle notations for permutations):

$$
\sigma_{1}=\left(\alpha_{3} \alpha_{4}\right)=\left(\delta_{3} \delta_{4}\right), \quad \sigma_{2}=\left(\alpha_{0} \alpha_{3}\right)=\left(\delta_{0} \delta_{3}\right), \quad \sigma_{3}=\left(\alpha_{1} \alpha_{4}\right)=\left(\delta_{1} \delta_{4}\right) .
$$

Then $\sigma_{i}$ act on the Picard lattice as

$$
\sigma_{1}=\left(\mathcal{E}_{6} \mathcal{E}_{8}\right) w_{\rho}, \quad \sigma_{2}=\left(\mathcal{E}_{3} \mathcal{E}_{7}\right)\left(\mathcal{E}_{4} \mathcal{E}_{8}\right), \quad \sigma_{3}=\left(\mathcal{E}_{1} \mathcal{E}_{5}\right)\left(\mathcal{E}_{2} \mathcal{E}_{6}\right)
$$

where $w_{\rho}$ is a reflection (A.8) in the root $\rho=\mathcal{H}_{f}-\mathcal{E}_{5}-\mathcal{E}_{7}$ (note also that a transposition $\left(\mathcal{E}_{i} \mathcal{E}_{j}\right.$ ) is induced by a reflection in the root $\mathcal{E}_{i}-\mathcal{E}_{j}$ ). The induced elementary birational mappings are then given by the following expressions: 
Proof. We briefly outline the proof for $\sigma_{1}$. First, note that we define the action of $\sigma_{1}$ on the symmetry roots $\alpha_{i}$ and then try to deduce its action on both the surface roots $\delta_{i}$ and also on all of Pic $(X)$. Moreover, $\sigma_{1}$ is an involution and from $\sigma_{1}: \alpha_{3} \leftrightarrow \alpha_{4}$ we see that it is natural to ask that $\sigma_{1}: \mathcal{H}_{f}-\mathcal{E}_{7} \leftrightarrow \mathcal{E}_{5}$ and $\sigma_{1}: \mathcal{E}_{6} \leftrightarrow \mathcal{E}_{8}$. Looking at the surface roots $\delta_{i}$ we see that then $\sigma_{1}$ permutes the roots $\delta_{3}$ and $\delta_{4}$, i.e., $\sigma_{1}: \mathcal{H}_{f}-\mathcal{E}_{5} \leftrightarrow \mathcal{E}_{7}$. Thus, $\sigma_{1}$ fixes $\mathcal{H}_{f}$. Requiring that $\sigma_{1}$ fixes $\alpha_{i}$ and $\delta_{i}$ for $i=0,1,2$ implies that $\sigma_{1}$ fixes $\mathcal{E}_{i}$ for $i=1, \ldots, 4$. From this it immediately follows that $\sigma_{1}\left(\mathcal{H}_{g}\right)=\mathcal{H}_{f}+\mathcal{H}_{g}-\mathcal{E}_{5}-\mathcal{E}_{7}$. It is now easy to see that $\sigma_{1}$ can be realized as a composition of two reflections in the roots $\mathcal{E}_{6}-\mathcal{E}_{8}$ and $\mathcal{H}_{f}-\mathcal{E}_{5}-\mathcal{E}_{7}$,

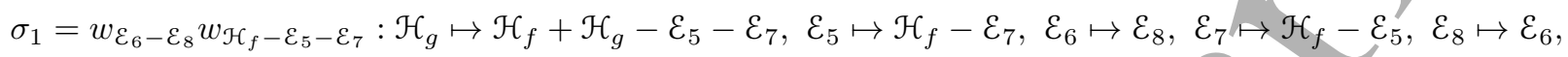

and the remaining generators of $\operatorname{Pic}(X)$ are fixed. The rest of the proof is now similar to the previous Theorem. Let $\sigma_{1}: X_{\mathbf{b}} \rightarrow X_{\overline{\mathbf{b}}}$ be written in the affine chart $(f, g)$ as $\sigma_{1}(f, g)=(\bar{f}, \bar{g})$. Requiring that

$$
\sigma_{1}^{*}\left(\mathcal{H}_{\bar{f}}\right)=\mathcal{H}_{f}, \quad \sigma_{1}^{*}\left(\mathcal{H}_{\bar{g}}\right)=\mathcal{H}_{f}+\mathcal{H}_{g}-\mathcal{E}_{5}-\mathcal{E}_{7}
$$

we get the mapping up to Möbius transformation,

$$
\bar{f}=\frac{A f+B}{C f+D}, \quad \bar{g}=\frac{K\left(g\left(f-b_{7}\right)+b_{5} b_{7}\right)+L f}{M\left(g\left(f-b_{7}\right)+b_{5} b_{7}\right)+N f}=\frac{K g(f-1)+L f}{M g(f-1)+N f},
$$

where we used the normalization $b_{5}=0$ and $b_{7}=1$; as usual, $A, \ldots, N$ are some constants to be determined. We also know that $\bar{a}_{3}=a_{4}, \bar{a}_{4}=a_{3}$, and $\bar{a}_{i}=a_{i}$ otherwise. Thus, $\bar{b}_{1}=b_{1}, \bar{b}_{2}=b_{2}, \bar{b}_{6}=b_{8}$, and $\bar{b}_{8}=b_{6}$. Note that $b_{3}=t$ is just a notation, it can (and actually will) evolve. From $\sigma_{1}\left(\mathcal{H}_{f}-\mathcal{E}_{5}\right)=\mathcal{E}_{7}$ we see that $(\bar{f}, \bar{g})(0, g)=\left(\frac{B}{D},-\frac{K}{M}\right)=(1, \infty)$ and so $B=D$ and $M=0$. From $\sigma_{1}\left(\mathcal{H}_{f}-\mathcal{E}_{7}\right)=\mathcal{E}_{5}$ we see that $(\bar{f}, \bar{g})(1, g)=\left(\frac{A+B}{C+D}, \frac{L}{M}\right)=(0,0)$ and so $A=-B$ and $L=0$. Finally, from $\sigma_{1}\left(\mathcal{E}_{1}\right)=\mathcal{E}_{1}$ we see that $(\bar{f}, \bar{g})\left(\infty, b_{1}\right)=\left(\frac{A}{C}, \frac{K b_{1}}{N}\right)=\left(\infty, b_{1}\right) ;$ so $C=0$ and $K=N$. Thus,

$$
\bar{f}=1-f, \quad \bar{g}=\frac{(f-1) g}{f} .
$$

Finally, from $\sigma_{1}\left(\mathcal{E}_{3}\right)=\mathcal{E}_{3}$ we see that $(\bar{f}, \bar{g})\left(b_{3}, \infty\right)=(\bar{f}, \bar{g})(t, \infty)=(1-t, \infty)=(\bar{t}, \infty)$, and so we see that the parameter $t$ indeed evolves, $\bar{t}=1-t$. This is related to the fact that elements from Aut $\left(D_{4}^{(1)}\right)$ are no longer standard Bäcklund transformations of $\mathrm{P}_{\mathrm{VI}}$. This completes the proof of (A.16). The proof for the other $\sigma \mathrm{s}$ is similar and is omitted.

Finally, the semi-direct product structure is defined by the action of $\sigma \in \operatorname{Aut}\left(D_{4}^{(1)}\right)$ on $W\left(D_{4}^{(1)}\right)$ via $w_{\sigma\left(\alpha_{i}\right)}=\sigma w_{\alpha_{i}} \sigma^{-1}$.

\section{A.4 The standard discrete d- $\mathrm{P}_{\mathrm{V}}$ Painlevé Equation}

As is well-known, there are infinitely many different discrete Painlevé equations of the same type, since they correspond to the non-conjugate translations in the affine symmetry sub-lattice $Q$. Some of these equations are special, since they either appear in applications, or have a particularly nice form, or have degenerations to other known equations. In the d-P $\left(\mathrm{D}_{4}^{(1)} / \mathrm{D}_{4}^{(1)}\right)$ family one such equation is known as a difference Painlevé-V equation, since it has a continuous limit to the differential Painlevé-V equation.

In [KNY17] this equation is given in the following form,

$$
\bar{f} f=\frac{t g\left(g-a_{4}\right)}{\left(g+a_{2}\right)\left(g+a_{1}+a_{2}\right)}, \quad g+\underline{g}=a_{0}+a_{3}+a_{4}+\frac{a_{3}}{f-1}+\frac{t a_{0}}{f-t},
$$

with the root variable evolution and normalization given by

$$
\bar{a}_{0}=a_{0}-1, \quad \bar{a}_{1}=a_{1}, \quad \bar{a}_{2}=a_{2}+1, \quad \bar{a}_{3}=a_{3}-1, \quad \bar{a}_{4}=a_{4}, \quad a_{0}+a_{1}+2 a_{2}+a_{3}+a_{4}=1 .
$$




\section{Acknowledgements}

AD acknowledges the support of the University of Northern Colorado Spring 2019 RSCW grant and the support of the MIMUW grant to visit Warsaw in August 2019; that visit was essential for the success of the project. GF acknowledges the support of the National Science Center (Poland) via grant OPUS 2017/25/B/BST1/00931. AS was supported by a University College London Graduate Research Scholarship and Overseas Research Scholarship.

Part of this work was done at the 15th International Symposium on Orthogonal Polynomials, Special Functions and Applications (OPSFA-19) in Hagenberg, Austria, and AD and GF would like to thank the organizers for an interesting and stimulating Conference.

We thank Kenji Kajiwara, Tomoyuki Takenawa, and Yasuhiko Yamada for helpful comments and discussions. We also thank the referees for valuable comments and suggestions.

\section{References}

[BG16] Alexei Borodin and Vadim Gorin, Lectures on integrable probability, Probability and statistical physics in St. Petersburg, Proc. Sympos. Pure Math., vol. 91, Amer. Math. Soc., Providence, RI, 2016, pp. 155-214. MR 3526828

[Bor03] Alexei Borodin, Discrete gap probabilities, and discrete Painlevé equations, Duke Math. J. 117 (2003), no. 3, 489-542. MR MR1979052 (2004g:39030)

[CDT17] Adrian Stefan Carstea, Anton Dzhamay, and Tomoyuki Takenawa, Fiber-dependent deautonomization of integrable 2D mappings and discrete Painlevé equations, J. Phys. A 50 (2017), no. 40, 405202, 41. MR 3708091

[Chi78] T. S. Chihara, An introductíon to orthogonal polynomials, Gordon and Breach Science Publishers, New York-London-Paris, 1978, Mathematics and its Applications, Vol. 13. MR 0481884

[Dom16] Diego Dominici, Polynomial sequences associated with the moments of hypergeometric weights, SIGMA Symmetry Integrability Geom. Methods Appl. 12 (2016), Paper No. 044, 18. MR 3492864

[DK19] Anton Dzhamay and Alisa Knizel, q-Racah polynomial ensemble and discrete q-Painlevé equations, Int. Math. Res. Not. IMRN (2019), no. doi:10.1093/imrn/rnz211, 1-47.

[DST13] Anton Dzhamay, Hidetaka Sakai, and Tomoyuki Takenawa, Discrete Schlesinger transformations, their Hamiltonian formulation, and Difference Painlevé equations, arXiv:1302.2972v2 [math-ph], 2013, pp. 1-29.

[DT18] Anton Dzhamay and Tomoyuki Takenawa, On some applications of Sakai's geometric theory of discrete Painlevé equations, SIGMA Symmetry Integrability Geom. Methods Appl. 14 (2018), no. $075,20$.

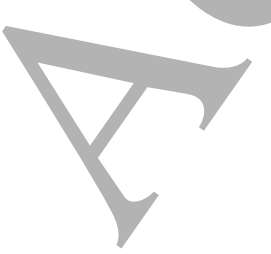


[FVA18] Galina Filipuk and Walter Van Assche, Discrete orthogonal polynomials with hypergeometric weights and Painlevé VI, SIGMA Symmetry Integrability Geom. Methods Appl. 14 (2018), Paper No. 088, 19. MR 3846851

[GR04] B. Grammaticos and A. Ramani, Discrete Painlevé equations: a review, Discrete integrable systems, Lecture Notes in Phys., vol. 644, Springer, Berlin, 2004, pp. 245-321. MR MR2087743 (2005g:39032)

[HFC19] J. Hu, G. Filipuk, and Y. Chen, Differential and difference equations for recurrence coefficients of orthogonal polynomials with hypergeometric weights and Bäcklund transformations of the sixth Painlevé equation, submitted, 2019.

[Ism05] Mourad E. H. Ismail, Classical and quantum orthogonal polynomials in one variable, Encyclopedia of Mathematics and its Applications, vol. 98, Cambridge University Press, Cambridge, 2005, With two chapters by Walter Van Assche, With a foreword by Richard A. Askey. MR 2191786

[KNY17] Kenji Kajiwara, Masatoshi Noumi, and Yasuhiko Yamada, Geometric aspects of Painlevé equations, J. Phys. A 50 (2017), no. 7, 073001, 164. MR 3609039

[NY98] Masatoshi Noumi and Yasuhiko Yamada, Affine Weyl groups, discrete dynamical systems and Painlevé equations, Comm. Math. Phys. 199 (1998), no. 2, 281-295. MR 1666847

[RGH91] A. Ramani, B. Grammaticos, and J. Hietarinta, Discrete versions of the Painlevé equations, Phys. Rev. Lett. 67 (1991), no. 14, 1829-1832. MR 1125951 (92j:39011)

[Sak01] Hidetaka Sakai, Rational surfaces associated with affine root systems and geometry of the Painlevé equations, Comm. Math. Phys. 220 (2001), no. 1, 165-229. MR MR1882403 (2003c:14030)

[Sha13] Igor R. Shafarevich, Basic algebraic geometry 1, Third ed., Springer, Heidelberg, 2013, Varieties in projective space. MR 3100243

[Sho39] J. Shohat, A differential equation for orthogonal polynomials, Duke Mathematical Journal 5 (1939), no. 2, 401-417.

[SKKT00] Karen E. Smith, Lauri Kahanpää, Pekka Kekäläinen, and William Traves, An invitation to algebraic geometry, Universitext, Springer-Verlag, New York, 2000. MR 1788561

[Sze67] Gábor Szegö, Orthogonal polynomials, third ed., American Mathematical Society, Providence, R.I., 1967, American Mathematical Society Colloquium Publications, Vol. 23. MR 0310533

[VA18] Walter Van Assche, Orthogonal polynomials and Painlevé equations, Australian Mathematical Society Lecture Series, vol. 27, Cambridge University Press, Cambridge, 2018. MR 3729446 